\title{
On the Variational Inequality Approach to Compressible Flows via Hodograph Method
}

\author{
LISA SANTOS
}

\begin{abstract}
We study the flow of a compressible, stationnary and irrotational fluid with wake, in a channel, around a convex symmetric profile, with assigned velocity $q_{\infty}$ at infinity and $q_{s}<q_{\infty}$ at the wake. In particular, we study the regularity of the free boundary (for a problem which has non-constant coefficients), in the hodograph plane.
\end{abstract}

Using variational inequalities, we obtain the solutions of some known cases as limit of this one, namely the solutions of the problems of an incompressible fluid in a channel without wake, in the plane with and without wake and the problem of a compressible fluid in the plane.

We also study the convergence of the free boundary of the new problem to the free boundaries of the limit corresponding problems.

\section{INTRODUCTION}

We study the flow with wake of a stationnary, irrotational, compressible fluid, with non-constant density $\varrho$, in a channel with semi-height $h$, around a convex symmetric profile, with given velocity $q_{\infty}$ at infinity and $q_{s}<q_{\infty}$ on the wake.

A change of variable of Baiocchi type in the physical formulation of the problem, after a hodograph transformation, leads us to a variational inequality; we also prove that its solution converges to the limit cases of a fluid

1991 Mathematics Subject Classification: 35J85.

Editorial Complutense. Madrid, 1993. 
-in the channel, without wake, when $q_{s} \rightarrow 0$,

$[(\varrho, h, 0)]$

-in the plane, with wake, when $h \rightarrow+\infty$,

$[(\varrho,+\infty, q)]$

-in the plane, without wake, when $h \rightarrow+\infty, q_{s} \rightarrow 0$,

$[(\varrho,+\infty, 0)]$

-incompressible, in each of the three situations

above, when the density $\varrho \rightarrow 1$,

$[(1, * * *)]$ being $*=h,+\infty$ and $* *=q_{s}, 0$.

The problem $(1,+\infty, 0)$ was studied by Brézis and Stampacchia ([7]), the problem $\left(1,+\infty, q_{s}\right)$ by Brézis and Duvaut $([5])$, the problem $(1, h, 0)$ by Tomarelli $([21])$ and the problem $(\varrho,+\infty, 0)$ by Brézis ([4]). These problems were also regarded from the numerical point of view, namely by Bourgat and Duvaut ([3]) and by Bruch and Dormiani ([8]). These kind of problems were also studied by Díaz ([9]), Díaz and Dou ([10]), by Hummel ([13], for non-symmetric convex profiles) and extended by Shimborsky ([19], [20]) to plane channels, Venturi tubes and flow around a Joukowski airfoil.

We are going to extend the formulation of these problems to the compressible case $\left(\varrho, h, q_{s}\right)$, in a channel, with wake, establishing that each of the previous cases is a (variational) limit in the hodograph plane, of this more complex case. We shall also prove the convergence of the free boundaries. These results for the incompressible case can be found in [18] and some of the results for the compressible case were announced in [17]. A new result is the study of the convergence of the solution of the problem of a compressible fluid to the solution of the problem of the incompressible one, when the density of the fluid $\varrho$ becomes constant.

In section 2 we formulate the physical problem and introduce the hodograph transformation. In section 3, after a Baiocchi type change of variable ([2]) in the hodograph plane, we present the variational formulation of the problem, for which we know there exists a unique solution. In section 4 we study the regularity of the solution and free boundary of the solution of the variational inequality, concluding that it is possible to turn back to the physical plane and we establish the existence and uniqueness of solution of the physical problem. In section 5 we study, firstly the convergence of the solution of the problem with wake to the solution of the problem without wake, when the velocity at the wake goes to zero and afterwards the convergence of the solution of our problem to the solution of the problem in the plane, when the height of the channel becomes arbitrarily large. In section 6 we extend the convergence results of sections 4 and 5 to the free boundaries. In the last section we prove the convergence of the solution and free boundary of the compressible problem to the solution and free boundary of the incompressible problem, when the density of the fluid goes to one.

\section{FORMULATION OF THE PROBLEM}

Let $\mathscr{P}$ be a strictly convex profile with height $2 H$, symmetric with respect to the $O X$ axe. Consider the flow of a fluid around this profile in a channel of semi-height $h>H>0$. We suppose the flow is uniform at infi- 
nity, with assigned velocity $q_{\infty}$ parallel to the $O X$ axe. The fluid has a given density $\varrho$, being

$$
\varrho=g(q)
$$

where $q$ is the velocity of the fluid and $g$ is a non-increasing $C^{1}$ function, bounded below by a strictly positive number $m$.

Let $S$ be the (unknown) boundary of the wake. We assume $S$ is a line, decreasing when $x$ grows, which intersects the profile in its descending part. Let $G$ be the exterior of the profile and wake in the channel.

By symmetry reasons, it is enough to work in the region $y \geq 0$. We denote by $G^{+}$and $\mathscr{P}^{+}$the intersections of $G$ and $\mathscr{F}^{+}$with the region $\{y \geq 0\}$.

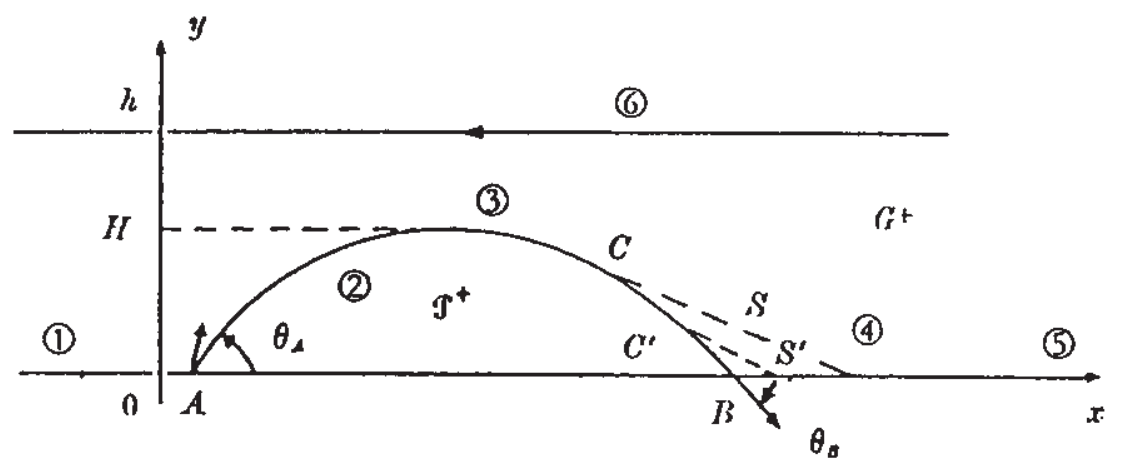

Fig. 1. The physical plane.

Let $\psi$ be the stream function defined as follows:

$$
\psi_{x}=-\varrho q_{2}, \quad \psi_{y}=\varrho q_{1} .
$$

Notice that $|\nabla \psi|=q g(q)$ and we can consider $\psi$ as a function of $x$ and $y$. Our problem can be formulated as follows:

$$
\begin{aligned}
& \psi \in C^{2}\left(G^{+}\right) \cap C^{1}\left(\overline{G^{+}}\right), \\
& \left(1-\frac{q_{1}^{2}}{a^{2}(q)}\right) \psi_{x x}+\left(1-\frac{q_{2}^{2}}{a^{2}(q)}\right) \psi_{y y}-2 \frac{q_{2} q_{2}}{a^{2}(q)} \psi_{x y}=0 \text { in } G, \\
& |\nabla \psi|=q_{s} g\left(q_{s}\right) \text { on } S, \\
& \psi(x, h)=h q_{\infty} g\left(q_{\infty}\right), \forall x \in \mathbb{R}, \\
& \psi=0 \text { on } \mathscr{P}+\cup S \text { or if } y=0, \\
& \lim _{|x| \rightarrow+\infty} \psi(x, y)=y q_{\infty} g\left(q_{\infty}\right), \text { uniformly in } y,
\end{aligned}
$$


being $a^{2}(q)=-q \frac{g(q)}{g^{\prime}(q)}$.

Consider now $\psi$ as function of $q$ and $\theta$, instead of $x$ and $y\left(q_{1}=q \cos \theta\right.$, $q_{2}=q \sin \theta$ ). Then

$$
q\left(\frac{1}{q g(q)}\right)_{q} \psi_{\theta \theta}=\left(\frac{q}{g(q)} \psi_{q}\right)_{q}
$$

Suppose that the equation $a(q)=q$ has a positive solution. Denote by $q_{c}$ the least positive solution of the equation $a(q)=q ; q_{c}$ is called the velocity of the sound (later on, we will allow $q_{c}=+\infty, a(q)=q$ has no positive solution).

Notice that the second equation of problem (2) is elliptic in the subsonic domain $\left(q<q_{c}\right)$ and hyperbolic in the supersonic domain $\left(q>q_{c}\right)$. Our study is restricted to the first case.

Define

and

$$
\sigma=\int_{q}^{q_{\varepsilon}} \frac{g(\tau)}{\tau} d r
$$

$$
\sigma_{\infty}=\int_{q_{\infty}}^{q_{c}} \frac{g(\tau)}{\tau} d \tau, \quad s=\int_{q_{s}}^{q_{s}} \frac{g(\tau)}{\tau} d \tau, \quad \sigma_{h}=\int_{q_{n}}^{q_{c}} \frac{g(\tau)}{\tau} d \tau,
$$

where $q_{h}=\max _{x \in \mathbb{R}}|\vec{q}(x, h)|$.

Assuming the fluid is totally subsonic, following Brézis ([4]), the profile $\mathscr{F}^{+}$is transformed in a curve $\mathscr{E}$ (which is a free boundary) contained in the region $\sigma>0$ that will be denoted by $\sigma=l(\theta)$.

Let $R(\theta)=-\left[\left(X^{\prime}\right)^{2}(\theta)+\left(Y^{\prime}\right)^{2}(\theta)\right]^{\frac{1}{2}}$ the curvature radius of ${\partial \mathscr{Y}^{+}}^{+}$on the point $(X(\theta), Y(\theta))$, being $(X(\theta), Y(\theta))$ the unique point $P$ of $\mathscr{Y}^{5}$ where the tangent at $P$ to $\partial \mathscr{\vartheta}^{+}$makes an angle $\theta(\theta<\pi / 2)$ with the axe $O X$. We suppose

$$
X, Y \in C^{1, \alpha}\left(\theta_{B}, \theta_{A}\right), \quad 0<\alpha<1 .
$$

We are going to work now in the hodograph plane, i.e., our coordinates are now $(\theta, \sigma)$ instead of $(x, y)$. We still denote our function, now on the variables $\theta$ and $\sigma$, by $\psi$. The figure below shows how the hodograph transformation acts. 


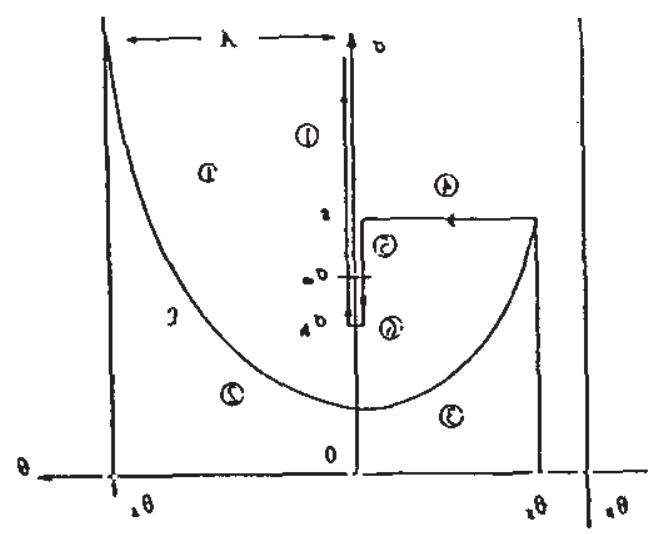

Fig. 2. The hodograph plane.

The angle between the wake and the profile in the point where both intersect is denoted by $\theta_{s}$. The region $G^{+}$is transformed in a region $\mathscr{Q}$, defined as follows:

$$
\begin{aligned}
\mathscr{Q}= & \left\{(\theta, \sigma): \theta_{s}<\theta<0, l(\theta)<\sigma<s\right\} \cup\left\{(\theta, \sigma): 0<\theta<\theta_{A}, l(\theta)<\sigma\right\} \\
& \cup\left\{(0, \sigma): l(0)<\sigma<\sigma_{h}\right\} .
\end{aligned}
$$

Since $\vec{q} \cdot \vec{n}=0$ on $S$, we conclude that $\psi \equiv 0$ on $S$.

Define

$$
\begin{gathered}
\left.\left.\left.\Omega_{s}=\right] \theta_{B}, 0\right] \times\right] 0, s[\cup] 0, \theta_{A}\left[\times \mathbb{R}^{+}, \quad \Gamma_{s}=\left\{(\theta, \sigma): \theta_{s}<\theta<0\right\},\right. \\
\Sigma_{h}=\left\{(0, \sigma): \sigma_{h}<\sigma<\sigma_{\infty}\right\}, \\
\Sigma_{\infty}=\left\{(0, \sigma): \sigma_{\infty}<\sigma<+\infty\right\} .
\end{gathered}
$$

Problem (2) is equivalent to the following one:

To find $\psi$ defined in $\Omega_{s}$ and $\mathscr{Q} \subseteq \Omega_{s}$ such that:

$$
\left\{\begin{array}{l}
k(\sigma) \psi_{\theta \theta}+\psi_{\sigma \sigma}=0 \text { in } \mathscr{S}, \\
\left\{\begin{array}{l}
\psi=0 \\
\psi_{\sigma}=-\frac{R(\theta) q(\sigma)}{1+k(\sigma) l^{\prime 2}(\theta)}
\end{array} \quad \text { on } \mathscr{L},\right. \\
\psi=0 \text { on } \Gamma_{s} \\
\psi=0 \text { on } \Sigma_{\infty}, \\
\psi=h q_{\infty} g\left(q_{\infty}\right) \text { on } \Sigma_{h}, \quad \theta>0 \\
\psi \rightarrow 0 \text { when } \sigma \rightarrow+\infty, \theta>0
\end{array}\right.
$$

being 


$$
k(\sigma)=\frac{1}{g^{2}(q(\sigma))}\left(1-\frac{q^{2}(\sigma)}{a^{2}(q(\sigma))}\right)
$$

\section{THE VARIATIONAL INEQUALITY}

In this section we assume the existence of a regular solution $\psi$ of the problem in the physical plane, verifying the following physically natural assumption:

«(*) The line in the physical plane, correspondent to $\theta=0$, intersects each line paralell to the axe $O X$ at most in one point».

Remark 3.1. Tomarelli established in [21] that the solution to the problem of the incompressible fluid in a channel verifies this property.

Let us make now a change of variable of Baiocchi type:

$$
u(\theta, \sigma)= \begin{cases}\int_{\mu(\theta)}^{\sigma} \frac{k(\tau)}{q(\tau)} \psi(\theta, \tau) d \tau & \text { if }(\theta, \sigma) \in \mathscr{Q} \\ 0 & \text { if }(\theta, \sigma) \in \Omega_{s} \backslash \mathscr{O}\end{cases}
$$

Proposition 3.2. The function $u$ verifies the following properties:

$$
\begin{aligned}
& u>0 \text { on } \mathscr{Q}, \frac{1}{q^{2}}\left(\frac{q^{2}}{k} u_{\sigma}\right)_{\sigma}+u_{\theta \theta}+u=-R(\theta) \text { on } \mathscr{Q}, \\
& u=u_{\theta}=u_{\sigma}=0 \text { on } \mathscr{C}, u_{\sigma}=0 \text { on } \Gamma_{s}, \\
& u(0, \sigma) \begin{cases}=H & \text { if } \sigma \geq \sigma_{\infty}, \\
=H-g\left(q_{\infty}\right) h q_{\infty} \int_{\sigma}^{\sigma_{\infty}} \frac{k(\tau)}{q(\tau)} d \tau & \text { if } \sigma_{h} \leq \sigma<\sigma_{\infty}, \\
\geq H-g\left(q_{\infty}\right) h q_{\infty} \int_{\sigma}^{\sigma_{\infty}} \frac{k(\tau)}{q(\tau)} d \tau & \text { if } l(0)<\sigma<\sigma_{h} .\end{cases}
\end{aligned}
$$

Proof. For the proof of the three first properties, see [4].

Since $u_{\sigma}=\frac{k(\sigma)}{q(\sigma)} \psi$, we have $u_{o}(\theta, s)=0$, and so $u_{\sigma}=0$ on $\Gamma_{s}$. 
If $\sigma \geq \sigma_{\infty}$, following [4] we conclude that $u(0, \sigma)=H$.

Notice that a point $(0, \sigma)$ of $\Sigma_{h}$ is image, by the hodograph transformation, of a point $(x, h)$, and $\psi(x, h)=h q_{\infty} g\left(q_{\infty}\right)$ (see (5)). If $\sigma$ is such that $l(0)<\sigma<\sigma_{h}, \quad(0, \sigma)$ is image of a point of $G^{+}$. As $\quad \psi=0 \quad$ on $\quad \partial \mathscr{P}^{+} \cup S \cup\{y=0\}, \quad \psi(x, h)=h g\left(q_{\infty}\right) q_{\infty} \quad$ and $\lim _{|x| \rightarrow+\infty} \psi(x, y)=y g\left(q_{\infty}\right) q_{\infty} \leq h g\left(q_{\infty}\right) q_{\infty}$, by the maximum principle (since $\psi$ satisfies an elliptic equation in $G^{+}$), we conclude that $\psi(0, \sigma)<h q_{\infty} g\left(q_{\infty}\right)$

Let $\sigma<\sigma_{\infty}$. Then

$$
\begin{aligned}
u(0, \sigma) & =\int_{l(0)}^{\sigma} \frac{k(\tau)}{q(\tau)} \psi(0, \tau) d \tau \\
& =\int_{l(0)}^{\sigma_{\infty}} \frac{k(\tau)}{q(\tau)} \psi(0, \tau) d \tau-\int_{\sigma}^{\sigma_{\infty}} \frac{k(\tau)}{q(\tau)} \psi(0, \tau) d \tau \\
& =H-\int_{\sigma}^{\sigma_{\infty}} \frac{k(\tau)}{\bar{q}(\bar{\tau})} \psi(0, \tau) d \tau,
\end{aligned}
$$

which concludes the proof.

Let $\left.\Gamma=] \theta_{B}, 0\right] \times\{s\}$. Define

$$
\begin{gathered}
V_{s}=\left\{v: q v \in L^{2}\left(\Omega_{s}\right), q v_{\theta} \in L^{2}\left(\Omega_{s}\right), \frac{q}{\sqrt{k}} v_{\sigma} \in L^{2}\left(\Omega_{s}\right),\left.v\right|_{\partial \Omega \backslash \Gamma_{s}} \equiv 0\right\}, \\
\|v\|_{V_{s}}^{2}=\int_{\Omega_{s}} q^{2}\left(\frac{1}{k} v_{\sigma}^{2}+v_{\theta}^{2}+v^{2}\right) d \theta d \sigma .
\end{gathered}
$$

Define on $V_{s}$ the following bilinear symmetric form

$$
a(u, v)=\int_{\Omega_{s}} q^{2}\left(\frac{1}{k} u_{o} v_{\sigma}+u_{\theta} v_{\theta}-u v\right) d \theta d \sigma
$$

and the convex set

$$
\mathbb{K}=\left\{v \in V_{s}: v \geq 0, v(0, \sigma)=H \text { if } \sigma \geq \sigma_{\infty}, v(0, \sigma) \geq \eta(\sigma) \text { if } \sigma<\sigma_{\infty}\right\},
$$

where

$$
\eta(\sigma)=H-h q_{\infty} g\left(q_{\infty}\right) \int_{\sigma}^{\sigma_{\infty}} \frac{k(\tau)}{q(\tau)} d \tau
$$


Lemma $3.3 a$ is coercive in $\mathbb{K}$, that is,

$$
\exists \alpha>0 \forall u, u_{0} \in \mathbb{K} a\left(u-u_{0}, u-u_{0}\right) \geq \alpha\left\|_{u}-u_{0}\right\|_{v_{s}^{\prime}}^{2}
$$

Proof. The proof is analogous to the one found in [4] for the problem in the whole plane, without wake, so we omit it.

Lemma $3.4 u \in \mathbb{K}$.

Proof. Results directly from Proposition 3.2.

\section{Lemma 3.5.}

$$
u_{\theta}\left(0^{-}, \sigma\right)-u_{\theta}\left(0^{+}, \sigma\right) \geq 0 \quad \text { if } \sigma_{h}<\sigma<\sigma_{\infty} .
$$

Proof. Reasoning as in [4], we conclude that

$$
u(\theta, \sigma)=\frac{1}{\varrho q} \psi+\sin \theta[x(\theta, \sigma)-X(\theta)]-\cos \theta[y(\theta, \sigma)-Y(\theta)]
$$

where $(x(\theta, \sigma), y(\theta, \sigma))$ is the point of the physical plane applied by the hodograph transformation in $(\theta, \sigma)$ and $(X(\theta), Y(\theta))$ is the parametrization of the profile indicated in the previous section.

Since

$$
\sin \theta=-\frac{1}{\varrho q} \psi_{x}, \quad \cos \theta=\frac{1}{\varrho q} \psi_{y}
$$

we conclude that

$$
u(\theta, \sigma)=\frac{1}{\varrho q}\left(\psi-x \psi_{x}-y \psi_{y}\right)-X(\theta) \sin \theta+Y(\theta) \cos \theta
$$

and as

$$
d\left(\psi-x \psi_{x}-y \psi_{y}\right)=-x d \psi_{x}-y d \psi_{y}
$$

we have 


$$
\begin{aligned}
u_{\theta}(\theta, \sigma) & =\frac{1}{\varrho q}\left[-x\left(\psi_{x}\right)_{\theta}-y\left(\psi_{y}\right)_{\theta}\right]+[-X(\theta) \sin \theta+y(\theta) \cos \theta]_{\theta}= \\
& =\frac{1}{\varrho q}[x \varrho q \cos \theta+y \varrho q \sin \theta]+[-X(\theta) \sin \theta+Y(\theta) \cos \theta]_{\theta}
\end{aligned}
$$

Then

$$
u_{\theta}\left(0^{-}, \sigma\right)-u_{\theta}\left(0^{+}, \sigma\right)=x\left(0^{-}, \sigma\right)-x\left(0^{+}, \sigma\right) .
$$

The property $\left(^{*}\right)$ imposed to the solution of the physical problem guarantees us that the line $\theta=0, l(0) \leq \sigma \leq \sigma_{h}$ in the hodograph plane corresponds, in the physical plane, to a one-to-one line that joins the unique point of the profile where $\theta=0$ (the point of the profile of maximum height), to a point of the line $y=h$ where the velocity is maximum. The complement of this curve in $G^{+}$has two connected components, one the set of the points where $\theta<0$ in the hodograph plane and the other the set of the points where $\theta>0$ in the hodograph plane. It is now obvious that $x\left(0^{-}, \sigma\right)-x\left(0^{+}, \sigma\right) \geq 0$.

Theorem 3.6 $u$ is the unique solution of the variational inequality

$$
\left\{\begin{array}{l}
u \in \mathbb{K}, \\
a(u, v-u) \geq \int_{\Omega_{s}} q^{2}(\sigma) R(\theta)(v-u) d \theta d \sigma, \quad \forall v \in \mathbb{K} .
\end{array}\right.
$$

Proof.

$$
\begin{aligned}
a(u, v-u)= & \int_{\Omega_{s}} q^{2}\left[\frac{1}{k} u_{\sigma}(v-u)_{\sigma}+u_{\theta}(v-u)_{\theta}-u(v-u)\right] d \theta d \sigma \\
= & \int_{\Xi} q^{2}\left[\frac{1}{k} u_{\sigma}(v-u)_{\sigma}+u_{\theta}(v-u)_{\theta}-u(v-u)\right] d \theta d \sigma \\
= & -\int_{s}\left\{\left(\frac{q^{2}}{k} u_{\sigma}\right)_{\sigma}(v-u)+q^{2} u_{\theta \theta}(v-u)\right. \\
& \left.+q^{2} u(v-u)\right\} d \theta d \sigma+\int_{\partial S}\left(u_{\theta}, \frac{q^{2}}{k} u_{\sigma}\right) \cdot \vec{n}(v-u),
\end{aligned}
$$

where $\partial \mathscr{Y}=\mathscr{Z} \cup \Sigma_{\infty} \cup \Sigma_{h} \cup \Gamma_{s}$ and $\vec{n}$ is the exterior normal vector to $\partial \mathscr{C}$.

Let $f(u, v)=\left(u_{\theta}, \frac{q^{2}}{k} u_{\sigma}\right) \cdot \vec{n}(u-v)$. On $\mathscr{Z}$ we have $u_{\theta}=u_{\sigma}=0$, so $\int_{\mathscr{Z}} f(u, v)=0$. On $\Sigma_{\infty}$, since $v(0, \sigma)=u(0, \sigma)=H$ we have $\int_{\Sigma_{x}} f(u, v)=0$. On $\Gamma_{s}, \vec{n}=(0,1)$ and $u_{\sigma}=0$, so $\int_{\Gamma_{1}} f(u, v)=0$. 
On $\Sigma_{h}, f(u, v)=\left(u_{\theta}\left(0^{-}, \sigma\right)-u_{\theta}\left(0^{+}, \sigma\right)\right)(v(0, \sigma)-u(0, \sigma))$. Using the previous lemma we know that $u_{\theta}\left(0^{-}, \sigma\right)-u_{\theta}\left(0^{+}, \sigma\right) \geq 0$. On the other hand, $v(0, \sigma) \geq \eta(\sigma)=u(0, \sigma)$ on $\Sigma_{h}$, so $\int_{\Sigma_{h}} f(u, v) \geq 0$.

Then

$$
\begin{aligned}
a(u, v-u) & \geq-\int_{\mathscr{Q}}\left(\left(\frac{q^{2}}{k} u_{\sigma}\right)_{\sigma}+q^{2} u_{\theta \theta}+q^{2} u\right)(v-u) d \theta d \sigma= \\
& =\int_{\mathscr{Q}} q^{2} R(\theta)(v-u) d \theta d \sigma
\end{aligned}
$$

by one of the properties of $u$ proved in Proposition 3.2. Since $v \geq 0$ in $\Omega_{s}, u \equiv 0$ in $\Omega_{s} \backslash \mathscr{Q}, R<0$, we have

$$
\int_{\Omega} q^{2} R(\theta)(v-u) d \theta d \sigma \geq \int_{\Omega} q^{2} R(\theta)(v-u) d \theta d \sigma,
$$

concluding then that

$$
a(u, v-u) \geq \int_{\Omega} q^{2} R(\theta)(v-u) d \theta d \sigma .
$$

The uniqueness of solution is a direct consequence of the fact that $a$ is a symmetric bilinear coercive form.

Remark 3.7. Related with this problem are the problems of flows of a compressible fluid with prescribed velocity $q_{\infty}$ at infinity, around a convex symmetric profile $\mathscr{P}$, in the three situations below:

(i) in a channel with semi-height $h$, without wake,

(ii) in the plane, with wake,

(iii) in the plane, without wake,

$$
\begin{aligned}
& {[(h, 0)],} \\
& {\left[\left(+\infty, q_{s}\right)\right],} \\
& {[(+\infty, 0)],}
\end{aligned}
$$

The physical formulation of these problems corresponds to omit in (2) the references to the wake, the channel or both.

Concerning the variational formulation, we have in each problem the variational inequality (15), being the space $V_{s}$ defined by (9) with the norm (10), being $s=+\infty$ and $\Gamma_{s}=0$ in the cases (i), (iii). The convex for each one of the three problems are the following ones:

- in case (i):

$$
\mathbb{K}_{h}=\left\{v \in V_{\infty}: v \geq 0, v(0, \sigma)=H \text { if } \sigma \geq \sigma_{\infty}, v(0, \sigma) \geq \eta(\sigma) \text { if } \sigma<\sigma_{\infty}\right\} ;
$$


- in case (ii):

$$
\mathbb{K}_{s}=\left\{v \in V_{\infty}: v \geq 0, v(0, \sigma)=H \text { if } \sigma \geq \sigma_{\infty}\right\} ;
$$

- in case (iii):

$$
\mathbb{K}_{\infty}=\left\{v \in V_{\infty}: v \geq 0, v(0, \sigma)=H \text { if } \sigma \geq \sigma_{\infty}\right\} .
$$

If we need to distinguish among the four diferent problems refered here, we put a subscript $\infty, s, h$ or $s h$ on the solutions, functions or convex sets related with the problems $(\infty, 0),\left(\infty, q_{s}\right),(h, 0)$ or $\left(h, q_{s}\right)$, respectively.

The problem treated here and in the next section is the case $\left(h, q_{s}\right)$ but all the results are easily ajustable to the other three cases.

\section{REGULARITY OF THE SOLUTION}

We are going to look now to (15) as a variational inequality in itself, independently of its origin, being $q(\sigma)$ and $k(\sigma)$ defined by

$$
\begin{gathered}
\frac{q^{\prime}(\sigma)}{q(\sigma)}=-\frac{1}{g(q(\sigma))}, q(\sigma)=\mathrm{T}, \\
k(\sigma)=\frac{1}{g^{2}(q(\sigma))}\left(1+\frac{q(\sigma) g^{\prime}(q(\sigma))}{g(q(\sigma))}\right),
\end{gathered}
$$

assuming from now on that $g \in W^{3, \infty}\left(0, q_{c}\right)$, in order to garantee the boundedeness of the first and second derivatives of $k$ and $q$. $q_{s}=q(s)$.

Notice that $\sigma_{\infty}$ is given and $q_{\infty}=q\left(\sigma_{\infty}\right) ; s$ is equally given, $s>\sigma_{\infty}$,

Since the variational inequality (15) has a unique solution we conclude that the physical problem has at most one solution physically natural, i.e., satisfying the property $\left({ }^{*}\right)$; if we prove that $u$ has enough regularity we can turn back to the physical plane, establishing the existence of a physically natural solution of the initial problem.

We begin with some important properties of the solution of (15), denoted in this section just by $u$, omiting the subscripts, since there is no risk of confusion.

Proposition 4.1. Let $u$ be the solution of (15). Then 


$$
\begin{gathered}
0 \leq u(\theta, \sigma) \leq H, \quad \forall(\theta, \sigma) \in \Omega_{s} \\
\|u\|_{V_{s}} \leq C, C \text { constant independent of } s \text { and } h .
\end{gathered}
$$

Proof. Let $v=u-(u-H \cos \theta)^{+}$. It is easy to verify that $v \in \mathbb{K}$ and $a(u-v, u-v) \leq 0$, using the fact that $u$ is solution of problem (15) and direct calculations. Then

$$
\left\|(u-H \cos \theta)^{+}\right\|^{2}=\|u-v\|^{2} \leq \frac{1}{\alpha} a(u-v, u-v) \leq 0,
$$

and so, $u \leq H \cos \theta \leq H$ in $\Omega_{s}$.

To prove (21) consider $\varphi \in C^{2}\left(\Omega_{s}\right) \cap V_{s}, \varphi(0, \sigma)=1$ if $\sigma \geq \sigma_{h}, 0 \leq \varphi \leq 1$, and let $w(\theta, \sigma)=H \varphi(\theta, \sigma)$. Obviously $w \in \mathbb{K}$.

Noticing that, for $w \in \mathbb{K}$ we have

$$
\|u\|^{2} \leq \frac{1}{\alpha} a(u, u)=\frac{1}{\alpha} a(u, w)-\frac{1}{\alpha} a(u, w-u)
$$

and

$$
-a(u, w-u) \leq-\int_{\Omega_{s}} R(\theta) q^{2}(w-u) d \theta d \sigma \leq-\int_{\Omega} R(\theta) q^{2} w d \theta d \sigma \leq C_{1},
$$

$C_{1}$ positive, since

$$
a(u, w) \leq \int_{\Omega_{s}} q^{2}\left(\frac{1}{k} u_{\mathrm{v}} w_{o}+u_{\theta} w_{\theta}+u w\right) \leq\|u\|\|w\|,
$$

we have

$$
\|u\|^{2} \leq \frac{C_{1}}{\alpha}+\frac{1}{\alpha}\|u\|\|w\|,
$$

concluding then that

$\|u\| \leq C, C$ depending only on $\alpha, C_{1}$ and $w$.

Define 


$$
\begin{gathered}
Q=\left\{(\theta, \sigma) \in \Omega_{s}: u(\theta, \sigma)>0\right\}, \quad(\Theta \text { is an open set }) ; \\
\theta_{*}=\inf \{\theta \in] \theta_{B}, 0[: u(\theta, s)>0\}, \\
\sigma_{*}=\inf \left\{\sigma: u(0, \sigma)=H-h q_{\infty} g\left(q_{\infty}\right) \int_{\sigma}^{\sigma_{\infty}} \frac{k(\tau)}{q(\tau)} \mathrm{d} \tau\right\}, \\
\Omega_{A}=\Omega_{s} \cap\{\theta>0\}, \quad \Omega_{B}=\Omega_{s} \cap\{\theta<0\}, \\
\Sigma_{\infty}=\{0\} \times\left[\sigma_{\infty},+\infty\left[, \Sigma_{*}=\{0\} \times\right] \sigma_{*}, \sigma_{\infty}\left[, \Gamma_{*}=\right] \theta_{*}, 0[\times\{s\} .\right.
\end{gathered}
$$
have:

Proposition 4.2. For every $\alpha, 0<a<1$ and some $\beta, 0<\beta<1$, we

$$
\begin{aligned}
& \text { a) } u \in C^{2, \alpha}\left(\mathscr{Q} \backslash \Sigma_{\infty} \cup \Sigma_{*} \cup \Gamma_{*}\right), \\
& u \in C^{1, a}\left(\Omega_{A} \cup \Sigma_{\infty}\right), u \in C^{1, \alpha}\left(\Omega_{B} \cup \Sigma_{\infty} \cup \Gamma_{*}\right) . \\
& \text { b) } u \in C^{1, \beta}\left(\Omega_{A} \cup \Sigma_{*}\right), u \in C^{1, \beta}\left(\Omega_{B} \cup \Sigma_{*}\right) .
\end{aligned}
$$

Proof. This proposition is a direct consequence of standard results on partial differential elliptic equations ([11], [14]), since $R \in C^{0, \alpha}\left(\theta_{B}, \theta_{A}\right)$, of a result of Grisvard ([12]) which establishes the $W^{2, p}$ regularity (for all $p$, $1 \leq p<+\infty$ ) near the corner $\Sigma_{\infty} \cap \bar{\Gamma}_{*}=\{(0, s)\}$ (for details see Lemma 5.1) and a result of Caffarelli for the Signorini problem ([11]).

Theorem 4.3. The free boundary $\mathscr{L}=\Omega_{s} \cap \partial\{(\theta, \sigma): u(\theta, \sigma)=0\}$ is a graph $\sigma=l(\theta)$ uniformly Lipschitz in $s$ and $h$, on any compact subset of ]$\theta_{*}, \theta_{A}\left[\right.$. The Lipschitz constant depends on $\|u\|_{C^{\prime}(K)}$, (being $K$ any compact subset of $\left.\Omega_{s}\right), \quad \max _{\theta \in\left[\theta_{k}, \theta_{A}\right]}|R(\theta)|, \quad \max _{\theta \in\left[\theta_{B}, \theta_{A}\right]}\left|R^{\prime}(\theta)\right|,\|q\|_{W^{2, \infty}}$ and on $\|k\|_{w^{2 . *}}$.

Proof. Since $\psi=\frac{q}{k} u_{\sigma}$ on $\Theta$, using that $\frac{1}{q^{2}}\left(\frac{q^{2}}{k} u_{\sigma}\right)_{\sigma}+u_{\theta \theta}+$ $u=-R(\theta)$, differentiating this equality in order to $\sigma$ and noticing that $\frac{k}{q}$ $=-\left(\frac{q_{o}}{q^{2}}\right)_{\sigma}$, we conclude that $k(\sigma) \psi_{\theta \theta}+\psi_{\sigma o}=0$ on $\mathscr{Q} \backslash\left(\Sigma_{\infty} \cup \Sigma_{*}\right)$. As $\psi=0$ on $\mathscr{L} \cup \Sigma_{\infty} \cup \Gamma_{*}$ and $\psi \geq 0$ on $\Sigma_{*}$, since on $\Sigma_{*}$ $u(0, \sigma)=H-h q_{\infty} g\left(q_{\infty}\right) \int_{\sigma}^{\sigma_{\infty}} \frac{k(\tau)}{q(\tau)} d r$, we conclude, by the strong maximum principle, that $\psi>0$ in $\mathscr{Q}$. Then $u_{\sigma}>0$ in $\mathscr{Q} \backslash\left(\Sigma_{\infty} \cup \Sigma_{*}\right)$ and 


$$
\forall \theta \in] \theta_{*}, \theta_{A}\left[3 ! \sigma_{\theta}: \quad u(\theta, \sigma)>0 \forall \sigma \geq \sigma_{\theta}\right.
$$

and we can define an upper semi-continuous parametrization of

$$
\left.l(\theta)=\inf \left\{\sigma \in \mathbb{R}^{+}: u(\theta, \sigma)>0\right\}, \forall \theta \in\right] \theta_{*}, \theta_{A}[.
$$

Observe that $l(0)<\sigma_{*}$, since $u_{o}\left(0, \sigma_{*}^{+}\right)>0$.

Let us verify now that $\mathscr{Z}$ does no contain vertical segments. Reasoning by contradiction, let $\gamma \subset \mathscr{Z}$ be such a segment. Then $\psi=0$ and $\psi_{\theta}=\frac{q}{k}\left(u_{\theta}\right)_{\sigma}=0$ on $\gamma$, since $u_{\theta}=0$ on $\mathscr{Z}$; on the other hand, each point of $\gamma$ is a point of minimum of the function $\psi$ and, by the Hopf maximum principle, $\frac{\partial \psi}{\partial n}= \pm \psi_{\theta}>0$ on $\gamma$ ( $n$ normal unitary vector), which is absurd.

To conclude that $l$ is Lipschitz in a neighborhood of $\left(\theta_{0}, \sigma_{0}\right) \in Z$, we are going to adapt to this case an argument of Alt ([1], [16]) for elliptic operators with constant coefficients. Let $L$ be the operator

$$
\begin{gathered}
L w=-\left[\frac{1}{q^{2}}\left(\frac{q^{2}}{k} w_{o}\right)+w_{\theta \theta}+w\right], \\
G=\{u>0\} \cap] \theta_{0}-\varrho, \theta_{0}+\varrho[\times] \sigma_{0}-\varrho, \sigma_{0}+\varrho\left[\subset \Omega_{s},\right. \\
F=\{(\theta, \sigma) \in] \theta_{0}-\varrho^{\prime}, \theta_{0}+\varrho^{\prime}[\times] \sigma_{0}-\varrho^{\prime}, \sigma_{0}+\varrho^{\prime}[: \sigma \geq l(\theta)\}, \varrho^{\prime}<\varrho,
\end{gathered}
$$

where $\varrho$ will be choosen conveniently, suficiently small (in particular in such a way that $\bar{G} \subset \Omega_{s}$ ).

Let $w=u+\varepsilon u_{\theta}-C \frac{1}{\sqrt{k}} u_{\theta}$. We will prove that

$$
\exists C_{0}>0 \forall C, C_{0} \leq C \leq 2 C_{0} \exists \varepsilon_{0}>0 \forall \varepsilon,|\varepsilon|<\varepsilon_{0} ; \leq \leq \text { in } F .
$$

Clearly we have

$$
L w=L u+\varepsilon(L u)_{\theta}-C L\left(\frac{l}{\sqrt{k}} u_{o}\right)
$$

Noticing that

$$
L\left(f u_{\sigma}\right)=f L u_{\sigma}-\frac{1}{q^{2}}\left(\frac{q^{2}}{k} f_{\sigma} u_{o}\right)_{\sigma}-\frac{f_{o}}{k} u_{\theta \theta}
$$


where $f=f(\sigma)$, and

$$
L u_{\sigma}=(L u)_{\sigma}+\left[\frac{1}{q^{2}}\left(\frac{q^{2}}{k}\right)_{\sigma}\right]_{\sigma} u_{\sigma}+\left(\frac{1}{k}\right)_{\sigma} u_{\sigma \sigma}
$$

we can verify that

$$
L\left(\frac{1}{\sqrt{k}} u_{\sigma}\right)=\left\{\frac{1}{q^{2}}\left[\frac{q^{2}}{k}\left(\frac{1}{\sqrt{k}}\right)\right]_{\sigma}-\frac{1}{q^{2}}\left[\frac{q^{2}}{k}\left(\frac{1}{\sqrt{k}}\right)\right]_{\sigma \sigma \sigma}\right\}_{\sigma}
$$

Let $-m$ be a lower bound $(m \geq 0)$ of

$$
\left\{\frac{1}{\sqrt{k}}\left[\frac{1}{q^{2}}\left(\frac{q^{2}}{k}\right)_{\sigma}\right]_{\sigma}-\frac{1}{q^{2}}\left[\frac{q^{2}}{k}\left(\frac{1}{\sqrt{k}}\right)_{\sigma}\right]_{\sigma}\right\}
$$

Then $L w \leq R(\theta)+\varepsilon R^{\prime}(\theta)+C m u_{\sigma}$.

Let

$$
\alpha=\max _{\theta \in\left[\theta_{B}, \theta_{A}\right]}\{R(\theta)\}<0
$$

Since $u_{o}=0$ on $\Gamma$, using the preceding proposition, we conclude that, given $C_{0}>0$, choosing $\varrho$ suficiently small, we have $2 \mathrm{C}_{0} m u_{\sigma} \leq-\alpha / 4$ in $G$. There exists also an $\varepsilon_{1}>0$ such that, if $|\varepsilon| \leq \varepsilon_{1}$, then $\varepsilon R^{\prime}(\theta) \leq-\alpha / 4$. So,

$$
\forall C, C_{0} \leq C \leq 2 C_{0} \quad \forall \varepsilon,|\varepsilon| \leq \varepsilon_{1} \quad L w \leq \alpha / 2 .
$$

Fix a non-negative function $\zeta$ of class $C^{2}(G)$ such that $\xi=0$ in $F$ and $\zeta \geq 1$ on $\partial G \backslash\left(\partial \mathscr{Q} \cap \Omega_{s}\right)$. Choose $\mu>0$ (that will depend only on $L, \alpha, \varrho$ and $\varrho^{\prime}$, and consequently on $\|q\|_{W^{2, \infty}}$ and $\|k\|_{W^{2,-}}$ through $\varrho$ ) such that

$$
-\mu L \zeta \leq-\frac{a}{2}
$$

If we prove that

$$
w \leq \mu \text { on } \partial G \backslash(\partial \varrho \cap \Omega s,
$$

since $w=0$ in $\partial \mathscr{Q} \cap \Omega_{s}$, using the maximum principle, we conclude that $w-\mu \zeta \leq 0$ on $G$, establishing then (23).

To prove (24), recall that $u_{\sigma}>0$ in $\mathscr{Q}$ and define 


$$
\gamma(\varepsilon)=\inf \left\{\frac{1}{\sqrt{k(\sigma)}} u_{\sigma}(\theta, \sigma):(\theta, \sigma) \in G \text { and } u(\theta, \sigma)>\varepsilon\right\} .
$$

Let us see that $\gamma(\varepsilon)>0$. Argue by contradition. Then there exists a sequence $\left(\xi_{n}\right)_{n}$ of elements of $G$ converging to an element $\xi_{0} \in G \cup \partial \Omega$ such that $\frac{1}{\sqrt{k\left(\sigma_{n}\right)}} u_{\sigma}\left(\xi_{n}\right) \rightarrow \gamma(\varepsilon) \leq 0$. Then we have $u\left(\xi_{0}\right) \geq \varepsilon$ and $u_{\sigma}\left(\xi_{0}\right)=\sqrt{k\left(\xi_{0}\right)} \gamma(\varepsilon) \leq 0$, which contradicts the fact that $u_{\sigma}$ is strictly positive in $\mathscr{G}$.

Let $M=\|u\|_{C^{\prime}(\bar{G})}$ and define

$$
\varepsilon_{0}=\min \left\{\frac{\mu}{1+M}, \varepsilon_{1}\right\}, \quad C_{0}=\frac{M}{\gamma\left(\varepsilon_{0}\right)} .
$$

Let $(\theta, \sigma) \in \partial G \backslash\left(\partial \subseteq \cap \Omega_{s}\right)$ be such that $u(\theta, \sigma) \leq \varepsilon_{0}$. Then $w(\theta, \sigma) \leq(1+M) \varepsilon_{0} \leq \mu$.

Let $(\theta, \sigma)$ be now a point of $\partial G \backslash\left(\partial \Theta \cap \Omega_{s}\right)$ such that $u(\theta, \sigma)>\varepsilon_{0}$. Then we have $w \leq M+\varepsilon_{0} M-M \leq \mu$. We have then established (24) and from this we easily deduce that $F$ verifies the (uniform) cone property (or equivalently $\partial F$ is Lipschitz) since

$$
(-\varepsilon, C) \cdot \nabla u \geq 0 \quad \forall C \geq C_{0} a \quad \forall \varepsilon:|\varepsilon| \leq \varepsilon_{0},
$$

where $a$ is a positive lower bound of the function $\sqrt{k}$ in $\bar{G}$, which exists by the continuity and strict positivity of $k$ in $[0, b]$, for any $b$ such that $0<b<q_{c}$.

\section{Theorem 4.4.}

$$
l \in C_{l o c}^{1 . \alpha} \text { and } u \in C^{1, \alpha}(\cup \mathscr{L})
$$

Proof. These results are direct consequence of known theorems on the regularity of the free boundaries (once more since $R \in C^{0, a}$ ) and of Theorem 4.3 ([11], [14], [16]).

Observe that, established the regularity of the function $u$ it is easy to verify the possibility of returning to the physical plane through the inverse transformation of the hodograph and that $\psi=\frac{q}{k} u_{\sigma}$ is the unique physically natural solution of the initial problem, with the desired regularity. 


\section{THE CONVERGENCE OF THE SOLUTION TO THE COMPRESSIBLE LIMIT CASES}

In this section we are going to let $s \rightarrow+\infty$ firstly (it corresponds to let the velocity of the wake $q_{s} \rightarrow 0$ ) and prove that the solution of problem $\left(*, q_{s}\right)$ converges to the solution of problem $(*, 0)$, being $*=h,+\infty$. The proofs are presented just for the case $h<+\infty$, being the other case similar. The second step is to let $h \rightarrow+\infty$ and prove that the solution of problem $\left(+\infty, q_{s}\right)$ is the limit of the solutions of problems $\left(h, q_{s}\right), q_{s} \geq 0$. The proof presented here is just for the case $q_{s}>0$, since the other case is analogous.

\section{Lemma 5.1.}

$$
\left|\left(u_{s h}\right)_{\theta}\left(0^{-}, s\right)\right| \leq C,
$$

$C$ contant independent of $s$ and $h$.

Proof. Denote, to simplify, $u_{s h}$ by $u$.

Let $\varphi \in C^{\infty}(\mathbb{R})$ be uniformly Lipschitz, $0 \leq \varphi \leq 1$, and such that $\varphi \equiv 1$ if $s-\sigma<\varepsilon, \varphi \equiv 0$ if $\sigma<s-3 \varepsilon, \varepsilon$ fixed less than $\left(s-\sigma_{\infty}\right) / 4$. Let $Q=] \theta_{B}, 0[\times] \sigma_{\infty}, s[$ and $w=\varphi u$. Let

$$
L=\frac{1 \partial}{q^{2} \partial \sigma}\left(\frac{q^{2}}{k} \frac{\partial}{\partial \sigma}\right)+\frac{\partial^{2}}{\partial \theta^{2}}-I .
$$

As $L u=-R(\theta) \chi_{\{u>0\}}, w$ is the solution of problem

$$
\left\{\begin{array}{l}
L w=-\varphi R(\theta) \chi_{\{u>0\}}+\frac{1}{q^{2}}\left(\frac{q^{2}}{k} \varphi_{\sigma} u\right)_{\sigma}+\frac{1}{k} \varphi_{\sigma} u_{\sigma}, \\
w_{\sigma}=0 \text { on } \Gamma_{s} \cup \Gamma_{0}, \\
w=\varphi H \text { on } \Sigma_{0} \\
w=0 \text { on } \Sigma_{\theta_{s}}
\end{array}\right.
$$

where $\left.\Gamma_{0}=\right] \theta_{B}, 0\left[\times\left\{\sigma_{\infty}\right\}, \Sigma_{0}=\{0\} \times\right] \sigma_{\infty}, s\left[, \Sigma_{\theta_{B}}=\left\{\theta_{B}\right\} \times\right] \sigma_{\infty}, s[$.

Let

$$
f=-\varphi R(\theta) \chi_{\{u>0\}}+\frac{1}{q^{2}}\left(\frac{q^{2}}{k} \varphi_{\sigma} u\right)_{\sigma}+\frac{1}{k} \varphi_{\sigma} u_{\sigma}
$$


Using the properties of $k, q$ and $u$, we can easily conclude that $f \in L^{2}(Q)$ and so $w$, which is the solution of $L w=f$, belongs to $H^{2}(Q)$. But, as

$$
H^{2}(Q) \smile W^{1, p}(Q), \forall p<+\infty,
$$

we have $w \in W^{1, p}(Q)$ and $f \in L^{p}(Q), \forall p<+\infty$.

By a result of Grisvard ([12]), since the necessary compatibility conditions are verified on the vertices and interior of the rectangle $Q$, we can assert that $w \in W^{2, p}(Q)$ and

$$
\|w\|_{W^{2, n}(Q)} \leq\left(\|f\|_{L^{p}(Q)}+\|\varphi H\|_{W^{2-l i p, p\left(\Sigma_{0}\right)}}\right)
$$

Since $\|u\|_{V_{3}}$ is independent of $s$ and $h$, we conclude that $\|f\|_{L^{p}(Q)}$ is independent of $s$ and $h$ and consequently, $\|w\|_{W^{2, .}(Q)}$ is bounded independently of $s$ and $h$. Since $w=u$ if $\sigma>s-\varepsilon$, we have that the $C^{0, a}$ norm of $u_{\theta}$ is bounded in a neighborhood of $\left(0^{-}, s\right)$, independently of $s$ and $h$.

\section{Lemma 5.2.}

$$
\int_{\theta_{*}}^{0}\left|\left(\psi_{s h}\right)_{a}(\theta, s)\right| d \theta \leq C,
$$

$C$ constant independent of $s$ and $h$.

Proof. For simplicity, we omit the subscripts sh. Since $u_{o}=\frac{k}{q} \psi$, and

$$
\frac{1}{q^{2}}\left(\frac{q^{2}}{k} u_{\sigma}\right)_{\sigma}+u_{\theta \theta}+u=-R(\theta) \text { in } \mathscr{Q}
$$

we have

$$
(q \psi)_{\sigma}=\left(-R(\theta)-u_{\theta \theta}-u\right) q^{2} \text { on } \Theta
$$

On points of the form $(\theta, s), \psi \equiv 0$, so

$$
\psi_{\sigma}(\theta, s)=\left[-R(\theta)-u_{\theta \theta}(\theta, s)-u(\theta, s)\right] q(s) .
$$

Besides that 


$$
\psi_{\sigma}(\theta, s)=\lim _{h \rightarrow 0^{-}} \frac{\psi(\theta, \sigma+h)}{h} \leq 0,
$$

so

$$
\begin{aligned}
\int_{\theta_{*}}^{0}\left|\psi_{\sigma}(\theta, s)\right| d \theta & =\int_{\theta_{*}}^{0}-\psi_{\sigma}(\theta, s) d \theta \\
& \leq \int_{\theta_{*}}^{0} u(\theta, s) q(s) d \theta+\int_{\theta_{*}}^{0} u_{\theta \theta}(\theta, s) q(s) d \theta \\
& \leqq \frac{\pi}{2} H q(s)+u_{\theta}\left(0^{-}, s\right) q(s) \\
& \leqq \frac{\pi}{2} H+u_{\theta}\left(0^{-}, s\right),
\end{aligned}
$$

since $q$ is non-increasing and $q(0)=1$.

Extend now $u_{s h}$ to $\Omega=\Omega_{\infty}$ as follows:

$$
\tilde{u}_{s h}(\theta, \sigma)= \begin{cases}u_{s h}(\theta, \sigma) & \text { if }(\theta, \sigma) \in \Omega_{s}, \\ u_{s h}(\theta, \sigma) & \text { if }(\theta, \sigma) \in \Omega \backslash \Omega_{s}\end{cases}
$$

\section{Proposition 5.3.}

$$
\tilde{u}_{s h} \in \mathbb{K}_{h} \cap L^{\infty}(\Omega)
$$

and $\tilde{u}_{s h}$ is the unique solution of the variational inequality

$$
\begin{gathered}
a\left(\tilde{u}_{s h}, v-\tilde{u}_{s h}\right) \geq \int_{\Omega}[R(\theta)+ \\
\left.+\varphi_{s h}(\theta, \sigma)\right] q^{2}\left(v-\tilde{u}_{s h}\right) d \theta d \sigma, \quad \forall v \in \mathbb{K} \cap L^{\infty}(\Omega),
\end{gathered}
$$

being

$$
\left.\varphi_{s h}(\theta, \sigma)=\frac{1}{q(s)}\left(\psi_{s h}\right)_{\sigma}(\theta, s) \chi_{E_{*},} \quad E_{*}=\right] \theta_{*}, 0[\times] s,+\infty[
$$

Proof. Denote $\tilde{u}_{s h}$ by $\tilde{u}$ and $u_{s h}$ by $u$. We can easily check that 


$$
\tilde{u} \in C^{1, a}\left(\mathscr{Q} \cup E_{*} \backslash\left(\Sigma_{\infty} \cup \Sigma_{*}\right)\right), \tilde{u} \in C^{0, \alpha}\left(\Sigma_{\infty} \cup E_{*}\right),
$$

and also that $\tilde{u} \in \mathbb{K}_{h} \cap L^{\infty}(\Omega)$.

Let us evaluate $L \tilde{u}$. Let $(\theta, \sigma) \in E_{*}$; then

$$
\begin{aligned}
\left\{\frac{1}{q^{2}}\left(\frac{q^{2}}{k} \tilde{u}_{\sigma}\right)_{\sigma}+\tilde{u}_{\theta \theta}+\tilde{u}\right\}(\theta, \sigma) & =u_{\theta \theta}(\theta, s)+u(\theta, s) \\
& =-R(\theta)-\frac{1}{q(s)} \psi_{o}(\theta, s)
\end{aligned}
$$

Then

$$
L \vec{u}= \begin{cases}L u & \text { in } \mathscr{Q} \\ 0 & \text { in } \Omega \backslash\left(\Omega_{s} \cup E_{*}\right) \\ -R(\theta)-\frac{1}{q(s)} \psi_{\sigma}(\theta, s) & \text { in } E_{*} .\end{cases}
$$

Since $\left.\quad \partial\left(\mathscr{Q} \cup E_{*}\right)=\mathscr{L} \cup \Sigma_{\infty} \cup \Sigma_{*} \cup\left\{\theta_{*}\right\} \times\right] s,+\infty\left[, \frac{\partial \tilde{u}}{\partial n}=0 \quad\right.$ on

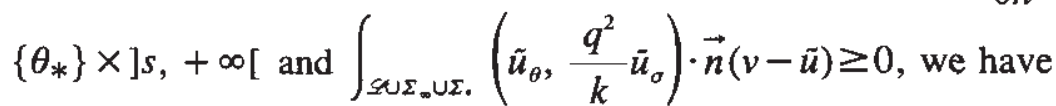

$$
\begin{aligned}
a(\tilde{u}, v-\tilde{u})= & \int_{\Omega} q^{2}\left\{\frac{1}{k} \tilde{u}_{\sigma}(v-\tilde{u})_{\sigma}+\tilde{u}_{\theta}\left(v-\tilde{u}_{\theta}\right)-\tilde{u}(v-\tilde{u})\right\} \\
= & -\int_{\mathscr{S} \cup E_{.}}\left\{\left(\frac{q^{2}}{k} \tilde{u}_{\sigma}\right)_{\sigma}(v-\tilde{u})+\tilde{u}_{\theta \theta}(v-\tilde{u}) q^{2}+\tilde{u}(v-\tilde{u}) q^{2}\right\} \\
& +\int_{\partial\left(G \cup E_{*}\right)}\left(\tilde{u}_{\theta}, \frac{q^{2}}{k} \tilde{u}_{\sigma}\right) \cdot \vec{n}(v-\tilde{u}) \\
\geq & \int_{\mathscr{S}} R(\theta) q^{2}(\sigma)(v-\tilde{u})+\int_{E_{*}}\left\{R(\theta)+\frac{1}{q(s)} \psi_{\sigma}(\theta, s)\right\} q^{2}(\sigma)(v-\tilde{u}) \\
\geq & \int_{\Omega}\left\{R(\theta)+\varphi_{s h}(\theta, \sigma)\right\} q^{2}(\sigma)(v-\tilde{u}),
\end{aligned}
$$

since $R \leq 0, \varphi_{s h} \leq 0,\left.\tilde{u}\right|_{\Omega \backslash\left(G \cup E_{.}\right)} \equiv 0$ and $v \geq 0$. 
Theorem 5.4.

$$
\left\|\tilde{u}_{s h}-u_{h}\right\|_{v} \leq C e^{-s} \quad \forall s>\sigma_{\infty},
$$

$C$ constant independent of $s$ and $h$.

In particular

$$
\tilde{u}_{s h} \underset{s \rightarrow+\infty}{\rightarrow} u \text { in } V \text {. }
$$

Proof. Recall that $\tilde{u}_{s h} \in \mathbb{K}_{h}$ and

$$
\exists \alpha>0 \quad \forall v, w \in \mathbb{K}_{h}:\|v-w\|_{v}^{2} \leq \frac{1}{\alpha} a(v-w, v-w) .
$$

Then

$$
\begin{aligned}
\alpha\left\|\tilde{u}_{s h}-u_{h}\right\|_{V}^{2} \leq & a\left(\tilde{u}_{s h}-u_{h}, \bar{u}_{s h}-u_{h}\right) \\
= & -a\left(\tilde{u}_{s h}, u_{h}-\tilde{u}_{s h}\right)-a\left(u_{h}, \tilde{u}_{s h}-u_{h}\right) \\
\leq & \int_{\Omega}\left\{R(\theta)+\varphi_{s h}(\theta, \sigma)\right\} q^{2}(\sigma)\left(u_{h}-\tilde{u}_{s h}\right) d \theta d \sigma \\
& -\int_{\Omega} R(\theta) q^{2}(\sigma)\left(\tilde{u}_{s h}-u_{h}\right) d \theta d \sigma \\
= & -\int_{\Omega} \varphi_{s h}(\theta, \sigma) q^{2}(\sigma)\left(u_{h}-\tilde{u}_{s h}\right) d \theta d \sigma \\
= & \int_{E_{*}}\left|\left(\psi_{s h}\right)_{\sigma}(\theta, \sigma)\right| \frac{q^{2}(\theta)}{q(s)}\left(u_{h}-\tilde{u}_{s h}\right) d \theta d \sigma \\
\leq & H \int_{\theta_{*}}^{0}\left|\left(\psi_{s h}\right)_{\sigma}(\theta, s)\right| d \theta \int_{s}^{+\infty} \frac{q^{2}(\sigma)}{q(s)} d \sigma,
\end{aligned}
$$

since $u_{h} \leq H$ and $\tilde{u}_{s h} \geq 0$.

As the function $q$ is decreasing since $q^{\prime}(\sigma)=-\frac{q(\sigma)}{g(q(\sigma))}<0$, we have $\frac{q(\sigma)}{q(s)}<1$ if $\sigma>s$. Using this fact and Lemma 5.2 we conclude that 


$$
\left\|\tilde{u}_{s h}-u_{h}\right\|_{v}^{2} \leq \frac{C_{1}}{\alpha} \int_{s}^{+\infty} q(\sigma) d \sigma
$$

$C_{1}$ constant independent of $s$ and $h$.

Since $q(\sigma) \leq e^{-\sigma}$, the estimation results directly from (32).

Extending now $u_{s}$ to $\Omega$ as we did with $u_{s h}$, it is possible to prove equally that $\left(u_{s}\right)_{\theta}\left(0^{-}, s\right)$ is bounded independently of $s, \tilde{u}_{s} \in \mathbb{K}_{\infty} \cap L^{\infty}(\Omega)$ and it is solution of a variational inequality analogous to (29). We have then

Theorem 5.5.

$$
\left\|\tilde{u}_{s}-u\right\|_{v}^{2} \leq C e^{-s}
$$

$C$ constant independent of $s$, and so

$$
\tilde{u}_{s} \rightarrow+\infty \text { in } V .
$$

Let us study now what happens when $h \rightarrow+\infty$, firstly with $s$ fixed. We have

Lemma 5.6. Let $u_{s \hat{h}}, u_{s h}$ and $u_{s}$ be the solutions of (15) with convex sets $\mathbb{K}_{s \hat{h}}, \mathbb{K}_{s h}$ and $\mathbb{K}_{s}$ respectively. Then

$$
u_{s \hat{h}} \geq u_{s h} \geq u_{s} \text { in } \Omega_{s}, \text { if } h<\hat{h} .
$$

Proof. Since the obstacle $\eta_{h}(\sigma)=H-h q_{\infty} g\left(q_{\infty}\right) \int_{\sigma}^{\sigma_{\infty}} \frac{k(\tau)}{q(\tau)} d \tau$ decreases on the segment $\{0\} \times \mathbb{R}^{+}$when $h$ increases, we have

$$
\mathbb{K}_{s \hat{h}} \subseteq \mathbb{K}_{s h} \subseteq \mathbb{K}_{s} \text { when } h<\hat{h} \text {. }
$$

and, using comparision arguments, we conclude (34).

\section{Theorem 5.7.}

$$
\left\|u_{s h}-u_{s}\right\|^{2} \leq C t_{h}^{1 / 2}, C \text { constant independent of } s \text { and } h,
$$


where $t_{h}$ is the unique solution $\eta_{h}\left(\sigma_{\infty}-\sigma\right)=0, \eta$ defined in (13).

Proof. Let

$$
W_{\infty}\left(\Omega_{s}\right)=\left\{v \in V_{s}: q v \in L^{\infty}, q v_{\theta} \in L^{\infty}, \frac{q}{\sqrt{k}} v_{\theta} \in L^{\infty}\right\},
$$

and suppose that

$$
\forall \cdot v \in \mathbb{K} \cap W_{. \infty}\left(\Omega_{s}\right) \quad \exists v_{h} \in \mathbb{K}_{h} \cap W_{\infty}\left(\Omega_{s}\right):\left\|v_{h}-v\right\|_{v_{s}}^{2} \leq C_{1} t_{h},
$$

$C_{1}$ constant independent of $s$ and $h$.

Since $\mathbb{K} \cap W_{\infty}\left(\Omega_{s}\right)$ is dense in $\mathbb{K}$, and $u_{s} \in \mathbb{K}$, there exists $v \in \mathbb{K} \cap W_{\infty}\left(\Omega_{s}\right)$ such that $\left\|v-u_{s}\right\|_{V_{s}}^{2} \leq C_{1} t_{h}$ and so

$$
\exists v_{h} \in \mathbb{K}_{h} \cap W_{\infty}\left(\Omega_{s}\right):\left\|u_{s}-v_{h}\right\|_{v_{s}}^{2} \leq 2 C_{1} t_{h^{*}}
$$

Then, since

$$
\begin{aligned}
\alpha\left\|u_{s h}-u_{s}\right\|_{v_{s}}^{2} & =a\left(u_{s h}-u_{s}, u_{s h}-u_{s}\right) \\
& =-a\left(u_{s}, u_{s h}-u_{s}\right)-a\left(u_{s h}, v_{h}-u_{s h}\right)-a\left(u_{s h}, u_{s}-v_{h}\right),
\end{aligned}
$$

and $a(u, v) \leq\|u\|\|v\|$ (as it was established in the proof of Proposition 21), we have

$$
\begin{aligned}
\alpha\left\|u_{s h}-u_{s}\right\|_{V_{s}}^{2} \leq & \int_{\Omega_{s}} R(\theta) q^{2}(\sigma)\left(u_{s h}-u_{s}\right) d \theta d \sigma \\
& +\iint_{\Omega_{s}} R(\theta) q^{2}(\sigma)\left(v_{h}-u_{s h}\right) d \theta d \sigma+\left\|u_{s h}\right\|_{V}\left\|u_{s}-v_{h}\right\|_{v_{s}} \\
\leq & \|R(\theta) q(\sigma)\|_{L^{2}\left(\Omega_{s}\right)}\left\|q(\sigma)\left(v_{h}-u_{s}\right)\right\|_{L^{2}(\Omega, s}+\left\|u_{s h}\right\|_{V_{s}}\left\|v_{h}-u_{s}\right\|_{v_{s}} \\
\leq & D\left\|v_{h}-u_{s}\right\|_{v_{s}}
\end{aligned}
$$

$D$ constant independent of $s$ and $h$.

Then

$$
\left\|u_{s h}-u_{s}\right\| \leq C t_{h}^{1 / 2}, C \text { constant independent of } s \text { and } h \text {. }
$$

To prove (36), given $v \in \mathbb{K} \cap W_{\infty}\left(\Omega_{s}\right)$, define $v_{h}(\theta, \sigma)$ as follows:

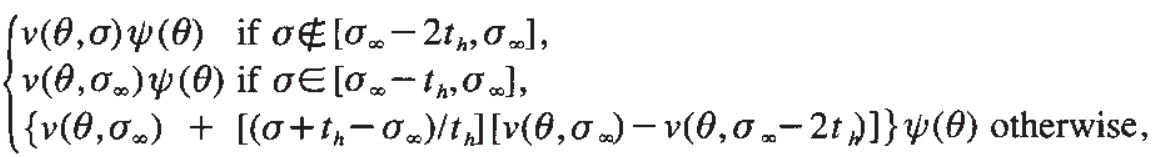


where $\psi \in C^{1}\left(\left[\theta_{B}, \theta_{A}\right]\right), \psi \geq 0, \psi(0)=1, \psi\left(\theta_{B}\right)=\psi\left(\theta_{A}\right)=0$.

Observe that $v_{h}(0, \sigma)=H$ if $\sigma \geq \sigma_{\infty}$.

If $\sigma \in\left[\sigma_{\infty}-t_{h}, \sigma_{\infty}\right], v_{h}(0, \sigma)=v(0, \sigma)=H \geq \eta_{h}(\sigma), \eta_{h}$ defined in (13).

If $\sigma \in\left[\sigma_{\infty}-2 t_{h}, \sigma_{\infty}-t_{h}\right], v_{h}(0, \sigma) \geq 0 \geq \eta_{h}(\sigma)$, since $t_{h}$ is the only solution of $\eta_{h}\left(\sigma_{\infty}-\sigma\right)=0$.

Since $v_{h} \in V_{s}, v_{h} \in \mathbb{K}_{h}$. Besides, $v_{h} \in W_{\infty}\left(\Omega_{s}\right)$.

Direct calculations show that

$$
\left\|v-v_{h}\right\|_{V_{s}}^{2} \leq C_{1} t_{h}, C_{1} \text { constant independent of } s \text { and } h .
$$

Remark 5.8. Since $k(\sigma) \neq 0$ in $\left[\frac{\sigma_{\infty}}{2}, \sigma_{\infty}\right]$ and $k$ is continuous,

$$
\exists \xi: k(\sigma) \geq \xi \quad \forall \sigma \in\left[\frac{\sigma_{\infty}}{2}, \sigma_{\infty}\right] .
$$

Then, for $t_{h}$ small enough,

$$
\int_{\sigma_{\infty}-t_{h}}^{\sigma_{\infty}} \frac{k(\tau)}{q(\tau)} d \tau \geqslant \xi e^{\sigma_{\infty}}\left(1-e^{-t_{h}}\right)
$$

using the fact that $q(\sigma) \leq e^{-\sigma}$. Then

$$
1-e^{-t_{h}} \leq \frac{H}{h q_{\infty} g\left(q_{\infty}\right) \xi e^{\sigma_{\infty}}}
$$

and, for $h$ big, we conclude that

$$
t_{h} \leq-\log \left(1-\frac{H}{q_{\infty} g\left(q_{\infty}\right) \xi e^{\sigma_{\infty}}} \frac{1}{h}\right) \sim \frac{C}{h} .
$$

Corollary 5.9. We have the following order of convergence

$$
\left\|u_{s h}-u_{s}\right\|_{V_{s}}^{2} \leq C h^{1 / 2}, C \text { constant independent of } s \text { and } h
$$

and consequently

$$
u_{s h} \underset{n \rightarrow+\infty}{\rightarrow} u_{s} \text { in } V_{s}
$$


Theorem 5.10.

$$
\left\|u_{h}-u\right\|_{V}^{2} \leq C t_{h}^{1 / 2}, C \text { constant independent of } h,
$$

being $t_{h}$ the unique solution of $\eta_{k}\left(\sigma_{\infty}-\sigma\right)=0, \eta_{h}$ defined in (13).

Furthermore

$$
\left\|u_{h}-u\right\|_{V}^{2} \leq C_{1} h^{-1 / 2}, C_{1} \text { constant independent of } h,
$$

and so,

$$
u_{h} \underset{h \rightarrow+\infty}{\rightarrow} u \text { in } V
$$

\section{STABILITY OF THE FREE BOUNDARIES}

Let

$$
\begin{array}{ll}
\mathscr{L}_{s h}=\partial\left\{u_{s h}=0\right\} \cap \Omega_{s}, & \mathscr{L}_{s}=\partial\left\{u_{s}=0\right\} \cap \Omega_{s}, \\
\mathscr{Z}_{h}=\left\{u_{h}=0\right\} \cap \Omega & \text { and } \quad \mathscr{L}=\{u=0\} \cap \Omega,
\end{array}
$$

be the free boundaries of problems $(s, h),(s,+\infty),(+\infty, s)$ and $(+\infty,+\infty)$. It is known, by Theorem 4.3 , that the free boundaries are graphs of functions $l_{s h}, l_{s}, l_{h}$ and $l$ respectively. Our aim in this section is to establish convergence results for these functions when $s \rightarrow+\infty$ or $h \rightarrow+\infty$.

We begin with the following lemma, for the problems with wake:

Lemma 6.1. $\theta_{*} \rightarrow \theta_{B}$ when $s \rightarrow+\infty$, being $\theta_{*}$ the angle beetwen the profile and wake on the point of intersection of both.

Proof. Since $\tilde{u}_{s h}$ are uniformly bounded in $W_{\mathrm{loc}}^{2, p}(\Omega), \tilde{u}_{s h} \underset{s \rightarrow+\infty}{\rightarrow} u_{h}$ in $W_{\text {loc }}^{2, p}$ - weak and, since $W^{2, p} \smile C^{1, \alpha}$, being the inclusion compact, we conclude that $\tilde{u}_{s h} \rightarrow u_{h}$ uniformly in the compact subsets of $\bar{\Omega}$. Since $l_{h}(\theta) \rightarrow+\infty$ when $\theta \rightarrow \theta_{B}$, we must have $\theta_{*} \rightarrow \theta_{B}$ when $s \rightarrow+\infty$.

Fix $\delta>0$ arbitrarily small. By Lemma 6.1

$$
\exists s_{\delta} \forall s \geq s_{\delta} \quad \theta_{*}<\theta_{B}+\delta / 2,
$$

(observe that $\theta_{*}=\theta_{*}(s)$ ). Let $I_{\delta}=\left[\theta_{B}+\delta, \theta_{A}-\delta\right]$. Then 
Theorem 6.2.

(1) $\left\|l_{s h}-l_{h}\right\|_{\left.c^{0, \alpha} a_{\delta}\right)} \leq C e^{-s(1-\alpha) / 4}, \quad \forall s \geq s_{\delta}$,

(2) $\left\|l_{h}-l\right\|_{C^{0 . a_{I_{\delta}}}} \leq \mathrm{Ch}^{-(1-\alpha) / 8}$,

(3) $\left\|l_{s h}-l_{s}\right\|_{C^{0, \alpha_{\left(I_{\delta}\right.}}} \leq C h^{-(1-\alpha) / 8}, \quad \forall s \geq s_{\delta}$,

(4) $\left\|l_{s}-l\right\|_{C^{0, a_{\left(I_{b}\right)}}} \leq C e^{-s(1-\alpha) / 4}, \quad \forall s \geq s_{\delta}$,

being $C$ constants, independent of $s$ and $h$ and $\alpha$ any number belonging to the interval $] 0,1[$.

Proof. The proof will be presented just for case (1), since the others are similar.

Let $\gamma \in C_{0}^{\infty}\left(\Omega \backslash\left(\Sigma_{*} \cup \Sigma_{\infty}\right)\right)$ be a cut off function such that $0 \leq \gamma \leq 1$, with $\gamma \equiv 0$ in a neighbourhood of $\left.\left(\mathscr{L}_{s h} \cup \mathscr{L}_{h}\right) \cap\right] \theta_{B}+\delta / 2, \theta_{A}-\delta / 2\left[\times \mathbb{R}^{+}\right.$ and supp $\gamma \subseteq \Omega_{s}$.

Then $\gamma \tilde{u}_{s h}$ is solution of the variational inequality

$$
a\left(\gamma \tilde{u}_{s h}, v-\gamma \tilde{u}_{s h}\right) \geq \int_{\Omega} \varphi_{1}(\theta, \sigma)\left(v-\gamma \tilde{u}_{s h}\right), \quad \forall v \in \mathbb{K},
$$

and $\gamma u_{h}$ is solution of the variational inequality

$$
a\left(\gamma u_{h}, v-\gamma u_{h}\right) \geq \int_{\Omega} \varphi_{2}(\theta, \sigma)\left(v-\gamma u_{h}\right), \quad \forall v \in \mathbb{K},
$$

being

$$
\begin{gathered}
\mathbb{K}=\{v \in V: v \geq 0\}, \\
\left.\varphi_{1}=\gamma q^{2}[R \theta)+\varphi_{s h}(\theta, \sigma)\right]-\left(\frac{q^{2}}{k} \gamma_{\sigma} u_{s h}\right)_{\sigma}-\gamma_{\sigma} \frac{q^{2}}{k}\left(u_{s h}\right)_{\sigma} \\
-q^{2} \gamma_{\theta \theta} u_{s h}-2 q^{2} \gamma_{\theta}\left(u_{s h}\right)_{\theta},
\end{gathered}
$$

and

$$
\varphi_{2}=\gamma q^{2} R(\theta)-\left(\frac{q^{2}}{k} \gamma_{\sigma} u_{h}\right)_{\sigma}-\gamma_{\sigma} \frac{q^{2}}{k}\left(u_{h}\right)_{\sigma}-q^{2} \gamma_{\theta \theta} u_{h}-2 q^{2} \gamma_{\theta}\left(u_{h}\right)_{\theta},
$$

being $\varphi_{s h}$ defined in (30).

Let $K=\gamma^{-1}(1)$. Since supp $\gamma$ is a compact set, $K$ is a compact subset of $\Omega_{s}$. Let 


$$
\beta=\min _{K}\left|R(\theta) q^{2}(\sigma)\right|>0 .
$$

Observe that

$$
\varphi_{1}=\varphi_{2}=q^{2}(\sigma) R(\theta) \leq-\beta<0, \text { on } K,
$$

so, using a result of [16], we have

$$
\begin{aligned}
\left\|l_{s h}(\theta)-l_{h}(\theta)\right\|_{L^{\prime}\left(\theta_{B}+\delta, \theta_{A}-\delta\right)} & \leq\left\|\chi_{1}-\chi_{2}\right\|_{L^{\prime}(K)} \\
& \leq \frac{1}{\beta} \int_{\Omega}\left|\varphi_{1}-\varphi_{2}\right| d \theta d \sigma,
\end{aligned}
$$

where $\chi_{1}=\chi_{\left\{u_{s h}=0\right\}}$ and $\chi_{2}=\chi_{\left\{u_{h}=0\right\}}$. But, since $q, q_{\sigma}$ and $k$ are bounded on $\dot{K}$ (notice that $(\theta, 0) \notin K$ ), we conclude that

$$
\begin{aligned}
\int_{\Omega}\left|\varphi_{1}-\varphi_{2}\right| & \leq \int_{\Omega_{s}} q^{2}\left|\gamma_{o}\left[\frac{q^{2}}{k}\left(u_{s h}-u_{h}\right)\right]_{\sigma}+2 \gamma_{\theta}\left(u_{s h}-u_{h}\right)_{\theta}\right|+\left|\gamma_{\theta \theta}\left(u_{s h}-u_{h}\right)\right| \\
& \leq C\left\|u_{s h}-u_{h}\right\|_{V}^{2} .
\end{aligned}
$$

We are going to use now the following interpolation inequality, due to Gagliardo-Nirenberg ([15]): for all $\alpha$ such that $0<\alpha<1$, for all $\varepsilon>0$,

$$
\|f\|_{C^{0, a}(a, b)} \leq C_{1} \varepsilon^{2(1+\alpha)}|| f^{\prime}\left\|_{L^{*}(a, b)}+\left(C_{2}+C_{1} \varepsilon^{2 /(\alpha-1)}\right)\right\| f \|_{L^{\prime}(a, b)},
$$

Since $l_{s h}-l_{h}$ is a Lipschitz function, with Lipschitz constant independent of $s$ and $h$, we have

$$
\begin{aligned}
\left\|l_{s h}-l_{h}\right\|_{C^{0, a}\left(\theta_{B}+\delta, \theta_{A}-\delta\right)} \leq & C_{1} \varepsilon^{2 /(1+\alpha)}\left\|l_{s h}^{\prime}-l_{h}^{\prime}\right\|_{L^{\alpha^{\prime}\left(\theta_{B}+\delta, \theta_{A}-\delta\right)}} \\
& +\left(C_{2}+C_{1} \varepsilon^{2(\alpha-1)}\right)\left\|l_{s h}-l_{h}\right\|_{L^{1}\left(\theta_{B}+\delta, \theta_{A}-\delta\right)} \\
\leq & C e^{-s(1-\alpha) / 4},
\end{aligned}
$$

choosing $\varepsilon=e^{-s(1-\alpha) / 4}$, and using Theorem 4.3 and property 42 .

\section{THE INCOMPRESSIBLE CASE AS A LIMIT CASE}

We are going to show in this section that, if $g_{n}$ is a sequence of density functions (of the fluid) such that

$$
\begin{gathered}
g_{n} \in W^{3, \infty}\left(\mathbb{R}_{0}^{+}\right), \\
\exists m, M>0 \quad \forall x \in \mathbb{R}^{+} \forall n \in \mathbb{N} \quad m \leq g_{n}(x) \leq M, \\
g_{n \rightarrow}^{\rightarrow} 1, g_{n}{ }^{\prime} \rightarrow 0 \text { uniformly on the compact subsets of } \mathbb{R}_{0}^{+},
\end{gathered}
$$


then, if $v_{n}$ is the solution of the problem (after a convenient translation) in one of the four situations refered before (in the channel with wake, in the channel without wake, in the plane with wake and in the plane without wake), with density function $g_{n}$, then $v_{n} \rightarrow v$ in a convenient space, being $v$ the solution of the limit problem with density function $g \equiv 1$; besides $e^{-a} v$ is the solution of the problem in the incompressible case, formulated as it appears in the literature.

Given $g_{n}$, define

$$
\xi_{n}=\int_{1}^{q_{c}^{n}} \frac{g_{n}(\tau)}{\tau} d \tau
$$

where $q_{c}^{n}$ denotes the velocity of the sound, which means the least solution of the equation $\frac{g_{n}(x)}{g_{n}^{\prime}(x)}=-x$. For $n$ sufficiently large, $\xi_{n}$ is positive, since $q_{c}^{n} \rightarrow+\infty$. Let

$$
s_{n}=\int_{q_{s}}^{1} \frac{g_{n}(\tau)}{\tau} d \tau, \quad \sigma_{n}=\int_{q_{\infty}}^{1} \frac{g_{n}(\tau)}{\tau} d \tau
$$

Let $q_{n}$ and $k_{n}$ be defined in $] 0,+\infty[$ by the following relations:

$$
\begin{gathered}
\frac{q_{n}^{\prime}(\sigma)}{q_{n}(\sigma)}=-\frac{1}{g_{n}\left(q_{n}(\sigma)\right)}, \quad q_{n}\left(\xi_{n}\right)=1, \\
k_{n}(\sigma)=\frac{1}{\left.g_{n}^{2}\left(q_{n}(\sigma)\right)\right)}\left(1+\frac{q_{n}(\sigma) g_{n}^{\prime}\left(q_{n}(\sigma)\right)}{g_{n}\left(q_{n}(\sigma)\right)}\right) .
\end{gathered}
$$

Recall that the variable $\sigma$ (which depends on $n$ ) is related with function $q_{n}$ (taken as a variable) by the following relation: $\sigma\left(q_{n}\right)=\int_{q_{n}}^{q_{c}^{n}} \frac{g_{n}(\tau)}{\tau} d \tau$. Redefining the problem with a different initial condition for $q_{n}$ corresponds to make a translation in the variable $\sigma$, more specificly, $\sigma \sim \sigma+\xi_{n}$, and, in the new variables, $\sigma\left(q_{n}\right)=\int_{q_{n}}^{1} \frac{g_{n}(\tau)}{\tau} d \tau$, the function $q_{n}$ evaluates 1 in zero and is defined on $]-\xi_{n},+\infty[$.

Let

$$
\left\{\begin{array}{l}
\left.\Gamma_{s_{n}}=\right] \theta_{B}, 0\left[\times\left\{s_{n}\right\}\right. \\
\left.\Omega_{s_{n}}=\right] \theta_{B}, 0[\times]-\xi_{n}, \mathbf{s}_{n}[\cup] 0, \theta_{A}[\times]-\xi_{n},+\infty[.
\end{array}\right.
$$


Observe that, from now on, everything done for $n \in \mathbb{N}$, has also a meaning for $n=\infty$, being, for simplicity, omited the subscript $\infty$ whenever convenient.

Let $n \in \mathbb{N} \cup\{+\infty\}$. The space $V_{n}$ in which we are going to work is

$$
\begin{gathered}
V_{n}=\left\{v: q_{n} v \in L^{2}\left(\Omega_{s_{n}}\right), q_{n} v_{\theta} \in L^{2}\left(\Omega_{s_{n}}\right),\right. \\
\left.\frac{q_{n}}{\sqrt{k_{n}}} v_{o} \in L^{2}\left(\Omega_{s_{n}}\right), v_{\mid \Omega_{s_{n}} \backslash s_{s_{n}}} \equiv 0\right\},
\end{gathered}
$$

with the canonical norm

$$
\|v\|_{n}^{2}=\int_{\Omega_{t}} q_{n}^{2}\left(\frac{1}{k_{n}} v_{\sigma}^{2}+v_{\theta}^{2}+v^{2}\right) d \theta d \sigma
$$

Let

$$
\zeta_{n}(\sigma)=H-h+\frac{h q_{\infty} g_{n}\left(q_{\infty}\right)}{q_{n}(\sigma) g_{n}\left(q_{n}(0)\right)}
$$

and

$$
\eta_{n}=\left(H \wedge \zeta_{n}\right) \vee 0 .
$$

The convex that appears in the variational formulation of the problem can be defined as follows:

$$
\begin{gathered}
\mathbb{K}_{n}=\left\{v \in V_{n}: v \geq 0, v(0, \sigma)=\eta_{n}(\sigma) \text { if } \sigma \geq \sigma_{n},\right. \\
\left.v(0, \sigma) \geq \eta_{n}(\sigma) \text { if } \sigma<\sigma_{n}\right\} .
\end{gathered}
$$

Consider the bilinear form

$$
a_{n}(u, v)=\int_{\Omega_{s_{n}}} q_{n}^{2}\left(\frac{1}{k_{n}} u_{\sigma} v_{\sigma}+u_{\theta} v_{\theta}-u v\right) d \theta d \sigma .
$$

The solution of the problem in the compressible case with density function $g_{n}$ is the unique solution $v_{n}$ of the problem

$$
\left\{\begin{array}{l}
v_{n} \in \mathbb{K}_{n}, \quad n \in \mathbb{N} \cup\{+\infty\}, \\
a_{n}\left(v_{n}, v-v_{n}\right) \geq \int_{n_{n}} R(\theta) q_{n}^{2}(\sigma)\left(v-v_{n}\right) d \theta d \sigma, \quad \forall v \in \mathbb{K}_{n^{*}}
\end{array}\right.
$$

We are going to verify now that all the problems (for any $n$ ) can be defined in the same open subset of $] \theta_{B}, \theta_{A}[\times \mathbb{R}$ and afterwards relate the limit problem $(n=+\infty)$ with the problem of the incompressible fluid. 
The following theorem (see [4]), gives us an a priori estimation of the maximum velocity of the fluid, or equivalently, of a lower bound of $\{\sigma: \exists \theta$ $v(\theta, \sigma)>0\}$, where $u_{n}$ is the solution of problem (56) with density function $g_{n}$. In the next theorem, since we are working in a fixed situation, we omit the $n$.

Theorem 7.1. Consider the problem (56) with density function $\mathrm{g}$. Let $\sigma_{*}=\inf \{\sigma: \zeta(\sigma)>0\}$, being $\zeta$ defined in (52), and $q\left(\sigma_{*}\right)=q_{*}$. Let $\alpha=$ $\min _{\theta \in\left[\theta_{B}, \theta_{A_{j}}\right.}|R(\theta)|>0$ and $q_{\mu}$ the least positive solution of the equation

$$
\frac{q_{\mu}}{q_{*}}\left[-1+\frac{1}{g\left(q_{*}\right)} \int_{q_{*}}^{q_{\mu}} \frac{g(\tau)}{\tau} \mathrm{d} \tau\right]=\frac{H}{\alpha}-1 .
$$

If $q_{\mu} \leq q_{c}$ then the maximum velocity of the fluid is less or equal to $q_{\mu}$.

Proof. Let $\mu=\int_{q_{\mu}}^{1} \frac{g(\tau)}{\tau} d \tau$ and define, for $\sigma \geq \mu$

$$
\varphi(\sigma)=\alpha q_{\mu} \int_{\mu}^{\sigma} \frac{k(\tau)}{q(\tau)}(\tau-\mu) d \tau .
$$

It is easily verified that $\varphi \geq 0, \varphi(\mu)=\varphi_{o}(\mu)=0$ and $q_{*} \leq q_{\mu}$. Besides, since $\frac{k}{q}=-\left(\frac{q_{\sigma}}{q^{2}}\right)_{\sigma}$, we conclude that

$$
\varphi(\sigma)=\alpha q_{\mu}\left[\frac{1}{q g(q)} \int_{q}^{q_{\mu}} \frac{g(\tau)}{\tau} d \tau-\frac{1}{q}+\frac{l}{q_{\mu}}\right] .
$$

Using (58) we conclude that $\varphi\left(\sigma_{*}\right)=H$. As $\varphi_{\sigma} \geq 0, \varphi \leq H$ if $\sigma \leq \sigma_{*}$. Extend $\varphi$ by zero to $\sigma<\mu$ and define $\Phi=\varphi \wedge H$. Simple calculations show that

$$
\frac{1}{q^{2}}\left(\frac{q^{2}}{k} \varphi_{\sigma}\right)_{o}+\varphi=\alpha
$$

Let $w=u-(u-\Phi)^{+}$, being $f^{+}=\max \{f, 0\}$. Observe that $w \in \mathbb{K}(\mathbb{K}$ defined in (54)). Then

$$
a\left(u,(u-\Phi)^{+}\right) \leq \int_{\Omega_{s}} R(\theta) q^{2}(\sigma)(u-\Phi)^{+}
$$


Let us evaluate now $a\left(\Phi,(u-\Phi)^{+}\right)$. Since $\Phi$ depends only on the variable $\sigma$ and $u(\theta,-\xi)=0$, integrating by parts we obtain

$$
\begin{aligned}
a\left(\Phi,(u-\Phi)^{+}\right) & =-\int_{\Omega_{s}}\left\{\left(\frac{q^{2}}{k} \Phi_{\sigma}\right)_{\sigma}+\Phi\right\}(u-\Phi)^{+} \\
& =\int_{\left\{\mu<\sigma<\sigma_{*} \mid\lceil(u>\Phi)\right.}-\alpha q^{2}(u-\Phi)^{+} \\
& \geq \int_{\Omega_{s}}-\alpha q^{2}(u-\Phi)^{+} .
\end{aligned}
$$

As it was refered before, there exists a positive constant $C$ such that $\left\|(u-\Phi)^{+}\right\|^{2} \leq C a\left(u-\Phi,(u-\Phi)^{+}\right)$and, since

$$
a\left(u-\Phi,(u-\Phi)^{+}\right) \leq \int_{\Omega_{s}}\{R(\theta)+\alpha\} q^{2}(u-\Phi)^{+} \leq 0,
$$

we conclude that $u \leq \Phi$ and, since $u \geq 0, u=0$ on $\{\sigma<\mu\}$.

Remark 7.2. The preceding theorem is valid whenever $q_{s} \leq q_{c}$. When $g_{n} \rightarrow 1$ and $g_{n}^{\prime} \rightarrow 0$ (uniformly on the compact sets), then $q_{c}^{n} \rightarrow+\infty$. On the other hand, observing equation (57) we easily verify that, if $q_{\mu}^{n}$ is the solution of (57) with density function $g_{n}, q_{\mu}^{n} \rightarrow q_{\mu}<+\infty, q_{\mu}$ solution of the same equation with density function $g \equiv 1$. Then, at least for $n$ big, the preceding theorem is true in our case.

We verify then that there exists $\mu \in \mathbb{R}$ such that, for $n$ big, $\mu>-\xi_{n}$ and $v_{n}(\theta, \sigma)=0$ for $\sigma \leq \mu$.

Consider the open set

$$
\left.\tilde{\Omega}_{s_{n}}=\right] \theta_{B}, 0[\times] \mu, s_{n}[\cup] 0, \theta_{A}[\times] \mu,+\infty[.
$$

Let $\vec{v}_{n}$ be the restriction of $v$ to $\tilde{\Omega}_{s_{n}}$. Define $\tilde{V}_{n}, \mathbb{K}$ and $\tilde{a}_{n}$ in the natural way, that is considering the intervenient functions with domain of definition $\tilde{\Omega}_{s_{n}}$. Then

Proposition 7.3. $\quad \tilde{v}_{n}$ is the unique solution of the variational inequality

$$
\left\{\begin{array}{l}
u_{n} \in \tilde{\mathbb{K}}_{n}, \quad n \in \mathbb{N} \cup\{+\infty\}, \\
\tilde{a}_{n}\left(u_{n}, v-u_{n}\right) \geq \int_{\dot{\Omega}_{s_{n}}} R(\theta) q_{n}^{2}(\sigma)\left(v-u_{n}\right) d \theta d \sigma, \quad \forall v \in \tilde{\mathbb{K}}_{n} .
\end{array}\right.
$$


Proof. The proof is immediate.

Remark 7.4. The formulation (62) of the problem will be the one used from now on; since there is no risk of confusion, we will omit the tilde on the functions, for simplicity of writing.

\section{Lemma 7.5.}

(a) $q_{n}(\sigma)=e^{-\int_{a}^{\sigma} \frac{d \tau}{g_{n}\left(q_{n}(\tau)\right)}}$

(b) $q_{n} \in C^{3}\left(\left[\mu,+\infty[)\right.\right.$ and $k_{n} \in C^{1}([\mu,+\infty[)$.

(c) $q_{n} \rightarrow e^{-\sigma}, q_{n}^{\prime} \rightarrow e^{-\sigma}$, uniformly on the compact subsets of $[\mu,+\infty[$ and $q_{n} \rightarrow e^{-\sigma}$ in $H^{1}(\mu,+\infty)$.

(d) $k_{n} \rightarrow 1, k_{n}^{\prime} \rightarrow 0$ uniformly on the compact subsets of $[\mu,+\infty[$ and $k_{n} \rightarrow 1$ in $H^{1}(\mu,+\infty)$.

Proof. These results are a direct consequence of the definitions of $q_{n}$ and $k_{n}$.

The limit problem is defined letting $n=\infty$ in problem (62). Notice that $q(\sigma)=e^{-\sigma}, k(\sigma)=1, \eta_{\infty}=\left(H \wedge \xi_{\infty}\right) \vee 0$, where $\xi_{\infty}(\sigma)=H-h+h q_{\infty} e^{\sigma}$.

The problem for an incompressible fluid (see [7]) has the following formulation: the open subset of $\mathbb{R}^{2}$ considered is

$$
\left.\left.\left.\Omega_{s}^{\prime}=\right] \theta_{B}, 0\right] \times\right] m, s[\cup] 0, \theta_{A}[\times] m,+\infty[,
$$

(see [16]) being $s=-\log \left(q_{s}\right)$ and $m$ any lower bound of $\tau_{h}+\sigma_{\infty}+\log (1-H /$ $h$ ), where $\tau_{h}$ is the unique negative solution of the equation

$$
\begin{gathered}
(1+H / \varrho)(1-H / h)=e^{\tau}(\tau+1+\log (1-H / h)) ; \\
\varrho=\max _{\theta \in\left(\theta_{g}, \theta_{A}\right]} R(\theta)<0 .
\end{gathered}
$$

Recall that $\left.\Gamma_{s}=\right] \theta_{B}, 0[\times\{s\}$. The space considered in this case is

$$
V^{\prime}=\left\{v \in H^{1}\left(\Omega_{s}^{\prime}\right): v_{\mid \partial \Omega_{s}^{\prime} \backslash Y_{s}} \equiv 0\right\},
$$


the convex set is

$$
\begin{gathered}
\mathbb{K}^{\prime}=\left\{v \in V^{\prime}: v \geq 0, v(0, \sigma)=H e^{-\sigma} \text { if } \sigma \geq \sigma_{\infty},\right. \\
\left.v(0, \sigma) \geq \Phi(\sigma) \text { if } \sigma<\sigma_{\infty}\right\},
\end{gathered}
$$

where $\Phi(\sigma)=h q_{\infty}+(H-h) e^{-\sigma}$, and the bilinear form is

$$
b(u, v)=\int_{\Omega_{s}^{\prime}} \nabla u \cdot \nabla v d \theta d \sigma+\int_{\Gamma_{s}} u v d \theta
$$

Then, the solution $u$ of the problem for an incompressible fluid, formulated in the hodograph plane, using variational inequalities, is the unique solution of

$$
b(u, v-u) \geq \int_{\mathrm{\Omega}_{s}} R(\theta) e^{-\sigma}(v-u), \quad \forall v \in \mathbb{K}^{\prime} .
$$

Obviously we can suppose $m=\mu$, extending $u$ or $v$ by zero, whether we are in the situation $m>\mu$ or in the other one. We need to extend the domain of definition of all functions and to make the appropriate change on the definition of the bilinear form. Since to $\mathbb{K}$ and $\mathbb{K}^{\prime}$ only belong nonnegative functions and $R(\theta)<0$, the extension of $u$ by zero is the solution of the extended problem.

Since, given $v, w \in V^{\prime}$,

$$
a\left(e^{\sigma} u, e^{\sigma} v\right)=\int_{\Omega_{s}}\left\{e^{2 \sigma}\left[\left(u v+(u v)_{\sigma}+u_{\sigma} v\right)+u_{\theta} v_{\theta}-u v\right]\right\} e^{-2 \sigma} d \theta d \sigma
$$

and

$$
\left.\left.\int_{\Omega_{s}}(u v)_{\sigma}=\int_{\theta_{z}}^{0} u v(\sigma)\right]_{\mu}^{s}+\int_{0}^{\theta_{A}} u v(\sigma)\right]_{\mu}^{+\infty}=\int_{r_{s}} u v d \theta,
$$

because, as $0 \leq u \leq H e^{-\sigma}, \lim _{\sigma \rightarrow+\infty} u(\theta, \sigma)=0$, we conclude that

$$
a\left(e^{\sigma} u, e^{\sigma} v\right)=b(u, v) \quad \forall u, v \in V^{\prime} .
$$

Since $e^{\sigma} v \in \mathbb{K}$ if and only if $v \in \mathbb{K}^{\prime}$, we conclude that $u$ is solution of (56) for $n=+\infty$ with the definition of $\Omega_{s}$ given in (61) if and only if $e^{-\sigma} u$ is the solution of (63).

Define, for $n \in \mathbb{N} \cup\{+\infty\}$,

$$
\hat{\mathbb{K}}_{n}=\left\{v \in V_{n}\left(\Omega_{s}\right): v \geq 0, v(0, \sigma)=\eta_{n}(\sigma) \text { if } \sigma \geq \sigma_{n},\right.
$$




$$
\left.v(0, \sigma) \geq \eta_{n}(\sigma) \text { if } \sigma<\sigma_{n}\right\},
$$

where $V_{n}\left(\Omega_{s}\right)$ has a definition analogous to $V_{n}$, being the domains of definition of the functions just restricted to $\Omega_{s}$. Being $v_{n}$ the solution of problem (56) we are going to define (by extension or restriction) $\hat{v}_{n} \in \hat{\mathbb{R}}_{n}$.

Suppose that $s_{n}>s$. Then define $\hat{v}_{n}=v_{n \mid \Omega_{s}}$. If $s \geq s_{n}$ extend $v_{n}$ to $\Omega_{s_{n}}$ as it was done in section 5 , that is

$$
\hat{v}_{n}(\theta, \sigma)= \begin{cases}v_{n}(\theta, \sigma) & \text { if }(\theta, \sigma) \in \Omega_{s_{n},} \\ v_{n}\left(\theta, \mathrm{s}_{n}\right) & \text { if }(\theta, \sigma) \in \Omega_{s}, \Omega_{s_{n}}\end{cases}
$$

Let $\quad \alpha=1 / m, m$ defined in

(45) and $W=W\left(\Omega_{s}\right)=\left\{v \in H^{1}\left(\Omega_{s}\right): v_{\mid \partial \Omega_{s} \backslash \Gamma_{s}} \equiv 0\right\}$. Let $Z=e^{-a \sigma} W$ with the canonical norm, that is, if $w \in Z,\|w\|_{Z}=\left\|e^{a \sigma} w\right\|_{H^{1}}$.

Theorem 7.6. If $v_{n}$ is the solution of problem (56) with density function $g_{n}$ and $v$ is the solution of the same problem with density function 1 and if the assumptions (44), (45) and (46) are verified, then

$$
\left\|\hat{v}_{n}-v\right\|_{Z}^{2} \rightarrow 0
$$

Proof. By Lemma 3.3, the bilinear form $a_{n}$ is coercive in

$$
K_{n}=\left\{v \in V_{n}\left(\Omega_{s}\right): v(0, \sigma)=H \text { if } \sigma>\sigma_{n}\right\} .
$$

Notice that $K_{n}$ contains $\hat{\mathbb{K}}_{n}$. Since $\left(q_{n}\right)_{n}$ and $\left(k_{n}\right)_{n}$ are sequences uniformly convergent in the compact subsets, the constant of coerciveness can be choosen independently of $n$, as we can verify in the proof of the refered lemma.

Since

$$
\exists a_{0}, b_{0}>0 \quad \forall \sigma \in[\mu,+\infty] \quad a_{0} \leq k_{n}(\sigma) \leq a_{1},
$$

and (45) is verified, we have that $V_{n}\left(\Omega_{s}\right)=q_{n}^{-1} W\left(\Omega_{s}\right)$ and

$$
\text { ㄱ. } \mathrm{C}_{1}, C_{2}>0 \quad \forall v \in V_{n} \quad C_{1}\left\|q_{n} v\right\|_{H^{1}} \leq\|v\|_{n} \leq C_{2}\left\|q_{n} v\right\|_{H^{1}},
$$

$C_{1}$ e $C_{2}$ constants independent of $n$.

Since $g_{n} \underset{n}{\rightarrow} 1$ uniformly on the compact sets, there exists $c_{n} \geqq 1, c_{n} \rightarrow 1$. So, $g_{n}(0) \leq c_{n}$ and, since $g_{n}$ is decreasing, $g_{n} \leq c_{n}$. Notice that $r_{n}=e^{-a / c_{n}}$ is the solution of equation (48) and $j_{n}=1 / c_{n}^{2}$ the solution of equation (49) 
(after translation of the variable $\sigma$ ) with $g_{n}$ substituted by $c_{n}$. Let $Z_{n}=r_{n}^{-1} W$ the canonical norm (that is, $\|w\|_{Z}=\left\|r_{n} w\right\|_{H^{1}}$ ). Then $Z_{n} \backsim \mathrm{V}_{n}$ and $\|v\|_{V_{n}} \leq C\|v\|_{Z_{n}}, C$ constant independent of $n$. Let $b_{n}$ be the following bilinear form, defined for $v, w \in Z_{n}$ :

$$
b_{n}(v, w)=\int_{\Omega} e^{-2 \sigma c_{n}}\left(c_{n}^{2} v_{o} w_{o}+v_{\theta} w_{\theta}-v w\right),
$$

and $w_{n}$ the solution of (56) with convex set $\hat{\mathbb{N}}_{n}$ defined as in (64), but for the functions $\tau_{n}$ and $j_{n}$.

To prove the result it is sufficient to establish that

$$
\left\|w_{n}-\hat{v}_{n}\right\|_{z \rightarrow} \rightarrow 0 \text { and }\left\|w_{n}-v\right\|_{z \rightarrow n} \rightarrow 0 \text {. }
$$

Recall that $\mathrm{Z}=r_{n}^{-1} W \subset V_{n}=q_{n}^{-1} W$ and $w_{n}(0, \sigma)=H$ for $\sigma \geq \sigma_{n}$, since $w_{n} \in \hat{\mathbb{M}}_{n}$.

It was proved in section 2 that $\left\|v_{n}\right\|_{v_{n}}$ are bounded independently of $n$ (and the same is verified by the extensions $\hat{v}_{n}$ ). Letting $\alpha$ be the constant of coerciveness of all the bilinear forms $a_{n}$ and $b_{n}$ (for $n$ big enough), we have

$$
\begin{aligned}
\left\|w_{n}-\hat{v}_{n}\right\|_{z}^{2} & \leq \frac{1}{\alpha} a_{n}\left(w_{n}-\hat{v}_{n}, w_{n}-\hat{v}_{n}\right) \\
& =\frac{1}{\alpha}\left[-a_{n}\left(\hat{v}_{n}, w_{n}-\hat{v}_{n}\right)-a_{n}\left(w_{n}, \hat{v}_{n}-w_{n}\right)\right] .
\end{aligned}
$$

Let $\psi \in C^{1}\left(\left[\theta_{B}, \theta_{A}\right]\right)$ verifying

$$
\psi \geq 0, \quad \psi\left(\theta_{B}\right)=\psi\left(\theta_{A}\right)=0, \quad \psi(0)=1
$$

and $\bar{w}_{n} \in q_{n}^{-1} W^{1, \infty} \cap V_{n}$ verifying $\left\|w_{n}-\bar{w}_{n}\right\|_{V_{n}} \leq \frac{1}{n}, \bar{w}_{n}(0, \sigma)=H$ if $\sigma \geq \sigma_{n}\left(\bar{w}_{n}\right.$ exists because $W^{1, \infty} \cap W$ is dense in $W$ ). Observe that $\left\|w_{n}-\tilde{w}_{n}\right\|_{v_{n} \rightarrow} 0$. Define

$$
\tilde{w}_{n}(\theta, \sigma)=\bar{w}_{n}(\theta, \sigma)+\left[\bar{w}_{n}(0, \sigma) \vee \eta_{n}(\sigma)-\bar{w}_{n}(0, \sigma)\right] \psi(\theta) .
$$

Easy verifications allow us to conclude that $\tilde{w}_{n} \in \mathbb{K}_{n} \cap q_{n}^{-1} W^{1, \infty}$. Notice that $\left\|\tilde{w}_{n}-w_{n}\right\| \|_{n \rightarrow \pi} 0$ and 


$$
\begin{aligned}
& -a_{n}\left(\hat{v}_{n}, w_{n}-\hat{v}_{n}\right)=-a_{n}\left(\hat{v}_{n}, \bar{w}_{n}-\hat{v}_{n}\right)-a_{n}\left(\hat{v}_{n}, w_{n}-\tilde{w}_{n}\right) \\
& \leq \int_{\Omega} q_{n}^{2}\left[R(\theta)+\varphi_{n}\right]\left(\hat{v}_{n}-\bar{w}_{n}\right)+\left\|\hat{v}_{n}\right\|_{V}\left\|w_{n}-\tilde{w}_{n}\right\|_{V_{n}}
\end{aligned}
$$

being $\varphi_{n}$ defined in (30).

Analogously we construct $z_{n} \in \hat{M}_{n} \cap r_{n}^{-1} W^{1, \infty},\left\|z_{n}-\hat{v}_{n}\right\|_{v_{n} \pi} 0$. Then

$$
\begin{gathered}
-a_{n}\left(w_{n}, \hat{v}_{n}-w_{n}\right) \leq \\
\left\|w_{n}\right\|_{V_{n}}\left\|\hat{v}_{n}-z_{n}\right\|_{V_{n}}+\int_{\Omega}\left\{r_{n}^{2}\left[R+\psi_{n}\right]\left(z_{n}-w_{n}\right)+\xi_{n}\left(w_{n}, z_{n}-w_{n}\right)\right\},
\end{gathered}
$$

being $\psi_{n}$ defined as $\varphi_{n}$, but for the problem with density of the fluid equal to $c_{n}$, and being $\xi_{n}=a_{n}-b_{n}$.

Then

$$
\begin{aligned}
\left\|w_{n}-\hat{v}_{n}\right\|_{Z}^{2} \leq & O(1)+\int_{\Omega_{s}} q_{n}^{2}\left[R+\varphi_{n}\right]\left(\tilde{w}_{n}-\hat{v}_{n}\right) \\
& +\int_{\Omega_{s}} r_{n}^{2}\left[R+\psi_{n}\right]\left(z_{n}-w_{n}\right) \\
= & \int_{\Omega_{s}} r_{n}^{2} R\left[\left(\tilde{w}_{n}-w_{n}\right)+\left(z_{n}-\hat{v}_{n}\right)\right] \\
& +\int_{\Omega_{s}}\left(q_{n}^{2}-r_{n}^{2}\right) R\left(\tilde{w}_{n}-w_{n}\right)+\int_{\Omega_{s} \backslash \Omega_{t_{n}}} \Phi_{n},
\end{aligned}
$$

where $\Phi_{n}=H\left(\left|\left(v_{n}\right)_{\theta}\left(0^{-}, s_{n}\right)\right|+\left|\left(w_{n}\right)_{\theta}\left(0^{-}, s_{n}\right)\right|\right)$ (for details, see calculations on section 3). Since $\left\|\vec{w}_{n}-w_{n}\right\|_{v_{n} \rightarrow} 0, q_{n}^{2}-r_{n}^{2} \rightarrow 0$ uniformly on the compact subsets of $[\mu,+\infty]$ and $\int \Omega_{s} \backslash \Omega_{s_{n}} \Phi_{n} \leq C\left|s-s_{n}\right|, C$ constant independent of $n$ (due to the uniform boundedeness of $v_{n}$ and $w_{n}$ in $C^{l, \alpha}\left(\Omega_{s_{n}}\right)$ ), we conclude that $\left\|w_{n}-\hat{v}_{n}\right\|_{z} \rightarrow 0$.

Analogously, we prove that $\left\|w_{n}-v\right\|_{Z \rightarrow} \rightarrow 0$, since $Z_{n}=r_{n}^{-1} W \subset V=e^{o} W$. Seeming this case simpler than the case treated before, it has an additional difficulty, since $\sigma_{n}$ changes, converging to $\sigma_{\infty}$ when $n \rightarrow+\infty$. Nevertheless, this difficulty can be easily solved, choosing the functions $\bar{w}_{n}$ in $q_{n}^{-1} \mathrm{~W}^{1, \infty} \cap V_{n}$ verifying $\tilde{w}_{n}(0, \sigma)=H$ for $\sigma \geq \sigma_{n} \wedge \sigma_{\infty}$.

We are going to study now the free boundaries. Recall that $g_{n} \in W^{3, \infty}$, and so the free boundaries are graphs of Lipschitz functions (with Lipschitz constants independent of $n$ ). 
The limit problem was not included in what was done in the preceding sections. However, we have seen in this section, after the translation of the coordinate $\sigma$ done above, that the limit problem is similar to the others and so, all the results previously obtained are also true for the limit problem.

Let $l_{n}$ and $l$ be parametrizations of $\partial\left\{u_{n}=0\right\} \cap \Omega$ and of $\partial\{u=0\} \cap \Omega$ respectively. We are going to prove the convergence of the free boundaries when $n \rightarrow+\infty$, which means that the free boundary of the problem of the compressible flow converges to the free boundary of the problem of the incompressible flow, when $g_{n} \rightarrow 1$.

Let us begin with a result of convergence of the angles of the wakes with the profile.

\section{Lemma 7.7.}

$$
\theta_{* \rightarrow n}^{n} \rightarrow \theta_{*},
$$

being $\theta_{*}^{n}$ the angle between the wake and the profile on the point of intersection of both, in the problem of the flow with density function $g_{n}$, and $\theta_{*}$ defined in the same way, but for the incompressible case.

Proof. Notice that

$$
\theta_{*}^{n}=\inf \left\{\theta: v_{n}\left(\theta, s_{n}\right)>0\right\}=\sup \left\{\theta: v_{n}\left(\theta, \mathrm{s}_{n}\right)=0\right\}
$$

Let us see that $\theta_{*}^{n}$ converges on the right for $\theta_{*}$. Suppose, by contradiction, that this does not happen, that is,

$$
\exists \varepsilon>0 \quad \forall p \in \mathbb{N} \quad \exists n_{p} \geq p \quad \theta_{*}^{n_{p}}>\theta_{*}+\varepsilon .
$$

Observe that $v_{n}\left(\theta, s_{n}\right) \rightarrow{ }_{n} v(\theta, s)$ uniformly, since $\hat{v}_{n}$ is uniformly bounded in $W_{l o c}^{2, p}\left(\Omega_{\infty}\right)$. Since $v_{n_{p}}\left(\theta, s_{n_{p}}\right)=0 \forall \theta<\theta_{*}^{n_{*}}$, we have $v_{n_{p}}\left(\theta, s_{n_{p}}\right)=0$, $\forall \theta<\theta_{*}+\varepsilon$ and so, passing to the limite, $v$ will be zero for all $(\theta, s)$ such that $\theta_{*}<\theta<\theta+\varepsilon$, which is against the definition of $\theta_{*}$.

Let us see now that $\theta_{*}^{n}$ converges on the left to $\theta_{*}$. Suppose not; then

$$
\text { э } \varepsilon>0 \quad \forall p \in \mathbb{N} \quad \exists n_{p} \geq p \quad \theta_{*}^{n_{p}}<\theta_{*}-\varepsilon .
$$

Fix $r, 0<r<\varepsilon$. Let $x_{0}=\left(\theta n_{*}^{n_{p}}, s_{n_{p}}\right)$ and notice that $x_{0} \in \bar{N}_{n_{p}}$, where $N_{n_{p}}=$ $\left\{(\theta, \sigma) \in \Omega_{s_{s_{p}}} ; v_{n_{p}}(\theta, \sigma)>0\right\}$. Then, by a result of Caffarelli, ([14]) 


$$
\sup _{B_{r}\left(x_{0}\right)}\left\{v_{n_{p}}(\theta, \sigma)-v_{n_{p}}\left(\theta_{*}^{n_{*},}, s_{n_{p}}\right)\right\} \geq \frac{\alpha}{4} r^{2},
$$

where $B_{r}\left(x_{0}\right)$ is the ball of center $x_{0}$ and radius $r$ and $\alpha=\min _{\theta}|R(\theta)|>0$.

Observe that $v_{n_{p}}\left(\theta_{*}^{n_{p}}, s_{n_{p}}\right)=0$ and, by the definition of least upper bound, there exists $\left(\theta_{n_{p}}, \sigma_{n_{p}}\right) \in B_{r}\left(x_{0}\right)$ such that $v_{n_{p}}\left(\theta_{n_{p}}, \sigma_{n_{p}}\right) \geq \frac{a}{8} r^{2}$. Since $v_{n_{p}}(\theta, \sigma)=v_{n_{p}}\left(\theta, s_{n_{p}}\right)$ if $\sigma>s_{n_{p}}$, and $\left(v_{n_{p}}\right)_{\sigma}(\theta, \sigma) \geq 0$, we conclude that $v_{n_{p}}\left(\theta_{n_{p}}, s_{n_{p}}\right) \geq v_{n_{p}}\left(\theta_{n_{p}}, \sigma_{n_{p}}\right)$. Since $\left(\theta_{n_{p}}, \sigma_{n_{p}}\right) \in B_{r}\left(x_{0}\right)$, we also have $\left(\theta_{n_{p}}, \mathrm{~s}_{n_{p}}\right) \in$ $B_{r}\left(x_{0}\right)$ and $\left|\theta_{n_{p}}-\theta_{*}^{n_{p}}\right|<r<\varepsilon$. Notice that $\theta_{n_{p}}<\theta_{*}^{n_{p}}+r<\theta_{*}+(r-\varepsilon)<\theta_{*}$ Since $\left(\theta_{n_{p}}\right)_{p \in \mathbb{N}}$ is a bounded sequence, it has a convergent subsequence to a number $\theta_{0}$ and $\theta_{0} \leq \theta_{*}+(r-\varepsilon)<\theta_{*}$. Since $v_{n_{p}}\left(\theta_{n_{p}}, s_{n_{p}}\right) \geq \frac{\alpha}{8} r^{2}$, passing to the limit we conclude that $v\left(\theta_{0}, s\right) \geq \frac{\alpha}{8} r^{2}$, which is in contradiction with the definition of $\theta_{*}$, since $\theta_{0}<\theta_{*}$.

Fix $\delta>0$ and let $p \in \mathbb{N}$ be such that

$$
\forall n \geq p \quad \theta_{*}^{n}<\theta_{*}+\delta .
$$

\section{Proposition 7.8.}

$$
\left\|l_{n}-l\right\|_{C^{0, a_{\left(\theta_{*}+\delta, \theta_{\beta}-\delta\right)}} \rightarrow} 0
$$

Proof. Let $\gamma$ be a cut off function with compact support such that $0 \leq \gamma \leq 1$, and $\gamma \equiv 1$ in a neighbourhood of $\left.\left(\mathscr{L}_{n} \cup \mathscr{L}\right) \cap\right] \theta_{B}+\delta / 2$, $\theta_{A}-\delta / 2\left[\times \mathbb{R}^{+}\right.$and such that supp $\gamma \subseteq \Omega_{s} \backslash \Sigma$, where $\Sigma=\{0\} \times\left[\inf _{n}\left\{\sigma_{n}\right\}\right.$, $+\infty[, n$ large enough.

Then, if $v_{n}$ is the solution of problem (56) and $v$ is the solution of the same problem for $n=+\infty, \gamma v_{n}$ is the solution of the variational inequality

$$
a_{n}\left(\gamma v_{n}, v-\gamma v_{n}\right) \geq \int_{\Omega_{s}} \xi_{n}(\theta, \sigma)\left(v-\gamma v_{n}\right), \quad \forall v \in \mathbb{K},
$$

and $\gamma \nu$ is the solution of the variational inequality

$$
a(\gamma v, v-\gamma v) \geq \int_{\mathrm{\Omega}_{s}} \xi(\theta, \sigma)(v-\gamma v), \quad \forall v \in \mathbb{K},
$$


where $\mathbb{K}=\{v \in V: v \geq 0\}$,

$$
\begin{aligned}
\xi_{n}= & \gamma q_{n}^{2}\left[R(\theta)+\varphi_{n}(\theta, \sigma)\right]-\left(\gamma_{\sigma} \frac{q_{n}^{2}}{k_{n}} v_{n}\right)_{\sigma}-\gamma_{\sigma} \frac{q_{n}^{2}}{k_{n}}\left(v_{n}\right)_{\sigma} \\
& -q_{n}^{2} \gamma_{\theta \theta} v_{n}-2 q_{n}^{2} \gamma_{\theta}\left(v_{n}\right)_{\theta},
\end{aligned}
$$

being $\varphi_{n} \equiv 0$ if $\Omega_{s} \subset \Omega_{s_{n}}$ and being $\varphi_{n}$ defined in (30) otherwise, and

$$
\xi=\gamma e^{-2 \sigma} R(\theta)-\left(\gamma_{\sigma} e^{-2 \sigma} v\right)_{\sigma}-\gamma_{\sigma} e^{-2 \sigma} v_{\sigma}-e^{-2 \sigma} \gamma_{\theta \theta} \nu-2 e^{-2 \sigma} \gamma_{\theta} \theta_{\theta} .
$$

Observe that

$$
\begin{aligned}
a\left(\gamma v_{n}, v-\gamma v_{n}\right) & =a_{n}\left(\gamma v_{n}, v-\gamma v_{n}\right)+\left(a-a_{n}\right)\left(\gamma v_{n}, v-\gamma v_{n}\right) \\
& \geq \int_{\Omega_{s}} \xi_{n}\left(v-\gamma v_{n}\right)-\int_{\Omega_{s}} \zeta_{n}\left(v-\gamma v_{n}\right), \quad \forall v \in \mathbb{K},
\end{aligned}
$$

where

$$
\begin{aligned}
\zeta_{n}= & {\left[\left(\frac{q_{n}^{2}}{k_{n}}-e^{-2 \sigma}\right)\left(\gamma v_{n}\right)_{\sigma}\right]_{\sigma}+\left[\left(q_{n}^{2}-e^{-2 \sigma}\right)\left(\gamma v_{n}\right)_{\theta \Theta}\right] } \\
& +\left[\left(q_{n}^{2}-e^{-2 \sigma}\right) \gamma v_{n}\right] .
\end{aligned}
$$

Let $K=\gamma^{-1}(1)$. Since $K$ is a compact subset of $\Omega_{s}$,

$$
a=d\left(K, \mathbb{R}^{2} \backslash \Omega_{s}\right)>0 \text { and } K \subset \Omega_{s-a / 2}
$$

and, since $s_{n} \rightarrow s$,

$$
\exists p \in \mathbb{N} \quad \forall n \geq p \quad s_{n}>s-a / 2,
$$

and so, $K \subset \Omega_{s_{n}}$, for $n \geq p$.

\section{Defining}

$$
\beta=\min _{K, n}\left\{q_{n}^{2}|R(\theta)|, \quad e^{-2 \theta}|R(\theta)|\right\}
$$

we have, in $K$,

$$
\left(\xi_{n}-\xi_{n}\right)(\theta, \sigma)=q_{n}^{2} R(\theta)-\left(q_{n}^{2}-e^{-2 \sigma}\right) \leq-\frac{\beta}{2}<0
$$

and 


$$
\xi(\theta, \sigma)=e^{-2 \sigma} R(\theta) \leq-\beta<0,
$$

for $n$ suficiently large, since $q_{n} \rightarrow e^{-\sigma}$ uniformly in $K$ (compact).

Using (just as for the stability of the free boundaries in $s$ and $h$ ) a result of [16], we conclude that

$$
\begin{aligned}
\left\|l_{n}-l\right\|_{L^{1}\left(\theta_{B}+\delta, \theta_{A}-\delta\right)} & =\left\|\chi_{n}-\chi\right\|_{L^{1}(K)} \\
& \leq \frac{2}{\beta} \int_{\Omega}\left|\left(\xi_{n}+\zeta_{n}\right)-\xi\right|
\end{aligned}
$$

where $\chi_{n}=\chi_{\left\{v_{n}=0\right\}}$ and $\chi=\chi_{\{v=0\}}$.

But

$$
\int_{\Omega}\left|\left(\xi_{n}-\xi_{n}\right)-\xi\right| \leq \int_{\Omega}\left|\xi_{n}-\xi\right|+\int_{\Omega}\left|\xi_{n}\right|
$$

Observe that

$$
\begin{aligned}
\int_{\Omega}\left|\xi_{n}-\xi\right| & \leq \int_{\Omega} \gamma\left|q_{n}^{2}-e^{-2 \sigma}\right||R(\theta)|+\int_{\Omega}\left|\left(\gamma_{\sigma} \frac{q_{n}^{2}}{k_{n}} v_{n}\right)_{\sigma}-\left(e^{-2 \sigma} \gamma_{\sigma} \nu\right)_{\sigma}\right| \\
& +\int_{\Omega}\left|\gamma_{\sigma} \frac{q_{n}^{2}}{k_{n}}\left(v_{n}\right)_{\sigma}-\gamma_{\sigma} e^{-2 \sigma} v_{\sigma}\right|+\int_{\Omega}\left|q_{n}^{2} \gamma_{\theta \theta} v_{n}-e^{-2 \sigma} \gamma_{\theta \theta} \nu\right| \\
& +\int_{\Omega}\left|2 q_{n}^{2} \gamma_{\theta}\left(v_{n}\right)_{\theta}-2 e^{-2 \sigma} \gamma_{\theta} v_{\theta}\right| \rightarrow \underset{n}{0} 0
\end{aligned}
$$

and that

$$
\int_{\Omega}\left|\zeta_{n}\right| \rightarrow 0
$$

since $q_{n} \rightarrow e^{-\sigma}, q_{n}^{\prime} \rightarrow-e^{-\sigma}, k_{n} \rightarrow 1$, and $k_{n}^{\prime} \rightarrow 0$ uniformly in the compact subsets of $\mathbb{R}, v_{n}$ and $v$ are bounded in $W_{p, l o c}^{2}$ (independently of $n$ ).

Since $l_{s}$ are Lipschitz function, by Theorem 4.3 , and the Lipschitz constants are independent of $n$, using the Gagliardo-Nirenberg inequality ([15]), we conclude, as in the preceding section that 


$$
\left\|l_{n}-l\right\|_{C^{0 . a_{\left(\theta_{*}+\delta, \theta_{A}-\delta\right)}} \rightarrow 0} 0
$$

\section{References}

[1] H. W. ALT: The fluid flow through porous media. Regularity of the free surface, Manuscripta Math. 305 (1977), 111-126.

[2] C. BAIOCCHI: Su un problema de fronteira libera conesso a questioni di idraulica, Ann. di Mat. Pura ed Appl. 92 (1972), 107-127.

[3] J. F. Bourgat and G. Duvaut: Numerical analysis of flow with or without wake past a symmetric two-dimensional profile without incidence, Inst. Journal for Num. Mat. in Eng. 11 (1977), 975-997.

[4] H. BREZIS: A new method in the study of subsonic flows, Lect. Notes Math. (Springer) (1975), 50-54.

[5] H. BRÉZIS and G. DUVAUT: Écoulements avec sillages autour d'un profil symetrique sans incidence, C. R. Acad. Sc. Paris, 276 (1973), 975-993.

[6] H. BRÉzis and G. STAMPACCHIA: Une nouvelle méthode pour l'étude d'écoulements stationnaires, C. R. Acad. Sc. Paris, 276, Serie A (1973), 129-132.

[7] H. BrézIs and G. StaMPaCCHIA: The hodograph method in fluid-dynamics in the ligth of variational inequalities, Arch. Rat. Mech. and Anal. 61 (1976), 1-18.

[8] J. BRUCH and M. DormianI: Flow past a symmetric two-dimensional profile with a wake in a channel, in "Nonlinear Problems», in Comput. Mech., vol. 2, edited by C. Taylor, O. R. Oden, E. Hinton, Pineridge Press, Swansea, U.K., 1987.

[9] J. I. DiAz: Técnica de supersoluciones locales para problemas estacionarios no lineales. Aplicación al estudio de flujos subsónicos, Real Acad. Cien. Madrid, XVI (1982).

[10] J. I. DiAZ and A. Dou: Sobre flujos subsónicos alrededor de un obstáculo simétrico, Collectanea Mathematica, 33 (1982), 141-160.

[11] A. FRIEDMAN: Variational principles and free boundary problems, WileyInterscience, New York, 1982.

[12] P. Grisvard: Équations differentiélles abstraictes, Ann. Scient. Éc. Norm. Sup. 42 (1969), 311-395.

[13] R. HUMMEL: The hodograph method for convex profiles, Ann. Scuo. Norm. Sup. Pisa, 9 IV (1982), 341-363.

[14] D. KINDERLEHRER and G. STAMPACCHIA: An introduction to variational inequalities and their applications, Academic Pres, New York, 1980.

[15] L. NiRENBERG: An extended interpolation inequality, Ann. Scuola Norm. Sup. Pisa, 20 (1966), 733-737.

[16] J. F. RoDRIGUES: Obstacle problems in mathematical Physics, North-Holland, Amsterdam, 1987.

[17] L. SANTOS: Asymptotic convergences in fluid dynamics-the compressible case, Pitman Res. Notes Math. Series \# 186 (1990), 788-792.

[18] L. SANTOS: Variational convergences of a flow with a wake in a channel past a profile, Bolletino U.M.I. 7 2-B (1988), 109-125.

[19] E. SHIMBORSKY: Variational methods applied to study of symmetric flows in laval nozzles: Commun. P. D. E. 4 (1979), 41-77. 
[20] E. SHIMBoRSKy: Variational inequalities arising in the theory of two dimensional potential flows: Nonlinear Anal. Theory Methods Appl. 5 (1981), 434-444.

[21] F. TOMARELl: Hodograph method and variational inequalities in fluid-dynamics, Ist. Nat. Alta Mat., Vol. I-II, Roma, 1980, 565-574.

[22] F. TOMARELLI: Un problème de fluidodynamics résolu avec les inéquations variationelles, C. R. Ac. Sci. Paris, 286 (1978), 999-1002.

Departamento de Matemática

Universidade do Minho

Campus de Gualtar

4700 Braga

Portugal

Recibido: 30 de diciembre de 1992 